\title{
Review Article \\ Recent Trends in Preparation of Poly(lactide-co-glycolide) Nanoparticles by Mixing Polymeric Organic Solution with Antisolvent
}

\author{
Edel Sah $^{1}$ and Hongkee Sah $^{2}$ \\ ${ }^{1}$ Department of Biological Sciences, University of Notre Dame, Notre Dame, IN 46556, USA \\ ${ }^{2}$ College of Pharmacy, Ewha Womans University, 52 Ewhayeodae-gil, Sedaemun-gu, Seoul 120-750, Republic of Korea \\ Correspondence should be addressed to Hongkee Sah; hsah@ewha.ac.kr
}

Received 19 September 2014; Revised 26 December 2014; Accepted 28 December 2014

Academic Editor: Mohamed Bououdina

Copyright (C) 2015 E. Sah and H. Sah. This is an open access article distributed under the Creative Commons Attribution License, which permits unrestricted use, distribution, and reproduction in any medium, provided the original work is properly cited.

In recent years, there have been a plethora of nanoengineering approaches for the development of poly(lactide-co-glycolide) (PLGA) nanoparticulate carrier systems. However, overlooking the multifaceted issues in the preparation and characterization of PLGA-based nanoparticles, many reports have been focused on their in vivo behaviors. It is imperative to fully assess technological aspects of a nanoencapsulation method of choice and to carefully evaluate the nanoparticle quality. The selection of a nanoencapsulation technique should consider drug property, nanoparticle quality, scale-up feasibility, manufacturing costs, personnel safety, environmental impact, waste disposal, and the like. Made in this review are the fundamentals of classical emulsiontemplated nanoencapsulation methods used to prepare PLGA nanoparticles. More specifically, this review provides insight into emulsion solvent evaporation/extraction, salting-out, nanoprecipitation, membrane emulsification, microfluidic technology, and flow focusing. Innovative nanoencapsulation techniques are being developed to address many challenges existing in the production of PLGA-based nanoparticles. In addition, there are various out-of-the-box approaches for the development of novel PLGA hybrid systems that could deliver multiple drugs. Latest trends in these areas are also dealt with in this review. Relevant information might be helpful to those who prepare and develop PLGA-based nanoparticles that meet their specific demands.

\section{Introduction}

Polymeric nanocarriers find versatile applications in drug solubilization, minimization of drug toxicity, improvement in drug stability, modulation of pharmacokinetics, sustained drug release, targeting, intracellular trafficking, and theranostics [1-5]. Natural and synthetic polymers are being explored as nanoparticle-forming materials [6]. Among them, the most widely used polymers in FDA-approved drug products and medical devices are poly(lactide-co-glycolide) and poly(lactic-co-glycolic acid) [7]. Hereinafter, these polymers are abbreviated as PLGA in text. PLGA-based drug products currently available in the market are implants and microspheres. Physicochemical property, biodegradation rate, and in vivo behavior of PLGA can be altered to fit a particular purpose by manipulating molecular weight, lactic acid : glycolic acid ratio, and end group. Diblock or triblock copolymers, such as PLGA-poly(ethylene glycol) (PEG), PLGA-PEG-PLGA, and PEG-PLGA-PEG, have also been developed to meet the need for better carrier functionality $[8,9]$.

There are many different emulsion-templated nanoencapsulation techniques used to prepare PLGA nanoparticulate carriers [10-12]. Representative examples are single or double emulsion solvent evaporation/extraction, nanoprecipitation, salting-out, membrane emulsification, microfluidic technology, and flow focusing. Each nanoencapsulation technique has distinct advantages and disadvantages. All these nanoencapsulation methods share a common feature of mixing a PLGA dispersed organic phase (in the form of either bulk or droplets) with antisolvent. Solvent removal is usually performed by evaporation, extraction, and/or combination of both. A newly coined nanoencapsulation term is often proposed to represent the principle of solvent removal. 
There are multifaceted issues that need to be considered in the preparation and development of PLGA nanoparticles via emulsion-templated nanoencapsulation methods. In the practice of conventional emulsification, the initial mixing of a polymeric dispersed phase in an aqueous phase takes place at a large scale. This bulk process makes it difficult to prepare homogeneous emulsion droplets and to precisely control the rate of PLGA precipitation. Common limitations arising with general mixing devices are lack of batch-tobatch reproducibility, wide variations in size distribution, and poor drug encapsulation efficiency. A heterogeneous nanoparticle population displays inconsistent behaviors in terms of systemic circulation, biodistribution, drug release, and cellular uptake. Therefore, it is hard to figure out which specific attribute of the nanoparticle population is held accountable for the event of study. There are other challenges in the production of PLGA-based nanoparticles. For example, the type of organic solvent used to dissolve PLGA presents critical issues in terms of emulsification, solvent removal, nanoparticle quality, and redispersibility after lyophilization. These critical attributes are also influenced by a mixing device used to disperse a polymeric dispersed phase in antisolvent. This review covers not only the fundamentals of various emulsion-templated nanoencapsulation methods but also the innovative approaches for meeting challenges in the production of PLGA nanoparticles.

\section{Classical Emulsion-Templated Nanoencapsulation Techniques}

2.1. Water-in-Oil (w/o) Emulsion Phase Separation/Coacervation. An aqueous drug solution is emulsified in an organic solution (e.g., methylene chloride) in which PLGA is dissolved. A phase inducer (e.g., silicone oil, vegetable oil, or mineral oil), being an antisolvent for PLGA but a solvent for the organic solvent, is added to the w/o emulsion to induce coacervation. Formation of coacervates around the inner aqueous phase and their fusion generate embryonic PLGA particulates. To harden them, the suspension is further treated with an organic solvent (e.g., heptane), which is a nonsolvent for PLGA but a solvent for the dispersed solvent and the phase inducer. EP 2131815 describes the principle of this process and its application in encapsulation of various watersoluble drugs into PLGA particles [13]. This method provides high encapsulation efficiencies toward hydrophilic drugs, due to their insolubility in organic solvents. However, controlling a coacervation step is quite difficult, and this method requires high costs of handling oils in large quantities.

2.2. Oil-in-Water (o/w) Emulsion Solvent Evaporation/Extraction. An o/w emulsion solvent evaporation method involves the following steps: (i) emulsification of a polymeric dispersed phase in an emulsifier-containing aqueous phase; (ii) solvent diffusion from emulsion droplets into the aqueous phase; (iii) solvent removal by evaporation; and (iv) solidification of emulsion droplets into PLGA nanoparticles. Solvent evaporation is usually carried out by continuous stirring at ambient pressure, but its rate can be accelerated under reduced pressure, vacuum, or elevated temperature. Methylene chloride, which has a low boiling point of $39.8^{\circ} \mathrm{C}$ and negligible water solubility $(1.32 \mathrm{wt} \%)$, is the preferred solvent of choice. This technique is particularly useful for loading hydrophobic drugs into PLGA nanoparticles.

Prior to emulsification, an emulsifier is added to a continuous phase and/or a PLGA organic solution. Common examples of surfactants and/or stabilizers are polyvinyl alcohol (PVA), didodecyldimethylammonium bromide, polyvinyl pyrrolidone, solutol, polysorbate, poloxamer, carbopol, polyethylene glycol (PEG), sodium dodecyl sulfate, proteins, carbohydrates, lecithin, and PEG-lipid (e.g., PEG-ceramide, $d$ - $\alpha$-tocopheryl polyethylene glycol 1000 succinate (TPGS)). It is important to notice that the type and concentration of surfactant affect the nanoparticle quality, drug encapsulation efficiency, drug release, pharmacokinetics, and cellular uptake/interaction. Therefore, a surfactant should be selected in consideration of the intended functionality and quality of PLGA nanoparticles.

In the practice of solvent extraction, an excessive amount of a quench liquid (i.e., water) is added into the o/w emulsion, in order to facilitate the quenching of the dispersed organic solvent into the aqueous phase. The amount of water used for solvent extraction usually exceeds at least 10 times the theoretical amount required for saturating water with the organic solvent. Ethyl acetate with a significant water miscibility $(\sim 8 \mathrm{wt} \%)$ is frequently used with this nanoencapsulation method. Solvent evaporation is often hybridized with solvent extraction to effectively remove organic solvents. Other treatments (e.g., dialysis, diafiltration/ultrafiltration, vacuum, and/or cosolvent treatment) are performed too. Table 1 summarizes experimental conditions used for nanoencapsulation of hydrophobic drugs and the major properties of PLGA nanoparticles. Important process parameters are PLGA, solvent, drug concentration, drug-polymer ratio, surfactant/ emulsifier, mixing device, organic phase-continuous phase ratio, solvent removal (method and rate), and freeze-drying condition (method and lyoprotectant).

\subsection{Oil-in-Oil $\left(o_{1} / o_{2}\right)$ Emulsion Solvent Evaporation/Extrac-} tion. A PLGA/drug/organic solvent dispersed phase $\left(o_{1}\right)$ is emulsified in a continuous oil phase (e.g., silicone oil, liquid paraffin, or vegetable oil; $\mathrm{o}_{2}$ ), and the process of solvent removal is carried out as mentioned earlier. Following this principle, Mahdavi et al. emulsified a PLGA/acetonitrile dispersed phase $\left(\mathrm{o}_{1}\right)$ in mineral oil $\left(\mathrm{o}_{2}\right)$ [35]. After solvent removal, PLGA nanoparticles were recovered by centrifugation and washed by $n$-hexane to eliminate residual mineral oil. The mean diameters and production yield of their nanoparticles were $640 \sim 890 \mathrm{~nm}$ and $38.5 \sim 45.3 \%$, respectively. Kashi et al. dissolved PLGA and minocycline in acetonitrile and emulsified the dispersed phase in Span 80containing mineral oil [36]. The resulting nanoemulsion was continuously stirred in $55^{\circ} \mathrm{C}$ for $2 \mathrm{hr}$ to remove acetonitrile by evaporation. Nanoparticles were collected by centrifugation and washed with $n$-hexane. The resultant particles had the mean diameter of $7.1 \mu \mathrm{m}$ and had an encapsulation efficiency of $5.5 \%$ for minocycline. Similarly, United States patent 
TABLE 1: Summary of processing conditions of o/w emulsion methods and major characteristics of PLGA nanoparticles.

\begin{tabular}{|c|c|c|c|c|c|c|c|}
\hline Polymer (grade) & Solvent & Polymer (\%) & Drug & $\mathrm{D} / \mathrm{P}$ & Size $(\mathrm{nm})$ & $\mathrm{EE}(\%)$ & Reference \\
\hline RG502H (0.16-0.24 dL/g) & DCM-DMSO & 2.5 & Amphotericin B & 5.6 & 154 & 53 & {$[14]$} \\
\hline PLGA50 : $50(40-75 \mathrm{kDa})$ & EA & 2 & Betamethasone-17-valerate & 60 & 281 & 85 & {$[15]$} \\
\hline mPEG-PLGA (11 kDa) & $\mathrm{DCM}$ & 2 & Bufalin & 5 & 164 & 82 & {$[16]$} \\
\hline RG502H (7-17 kDa) & $\mathrm{DCM}$ & 5 & $\beta$-Carotene & 10 & $200 \sim 800$ & 1 & {$[17]$} \\
\hline PLGA75 : 25 (66-107 kDa) & DCM-acetone & $0.1,0.2$ & Celecoxib & 25,50 & $115 \sim 436$ & $31 \sim 86$ & {$[18]$} \\
\hline PLGA50 : $50(10 \mathrm{kDa})$ & EA, PC & 2 & Coumarin- 6 & 5 & $56 \sim 306.6$ & $41 \sim 52$ & [19] \\
\hline RG $50: 50 \mathrm{H}(40-75 \mathrm{kDa})$ & EA-DCM & 2.5 & Cucumin & 10 & 162 & 77 & {$[20]$} \\
\hline RG 503 (n/a) & $\mathrm{DCM}$ & 1.67 & Cyclosporine A & 20 & 220 & 90 & {$[21]$} \\
\hline PLGA50 : $50(20-23 \mathrm{kDa})$ & $\mathrm{DCM}$ & 1 & Exemestane & $\mathrm{n} / \mathrm{a}$ & 169 & 64 & {$[22]$} \\
\hline PLGA50 : $50(0.36 \sim 037 \mathrm{dL} / \mathrm{g})$ & $\mathrm{DCM}$ & 3.3 & Haloperidol & $2.5 \sim 5$ & 524 & $23 \sim 37$ & [23] \\
\hline $\operatorname{PLGA}(\mathrm{n} / \mathrm{a})$ & $\mathrm{DCM}$ & $2.5 \sim 5.0$ & Nimesulide & $5 \sim 10$ & $212 \sim 289$ & $25 \sim 100$ & {$[24]$} \\
\hline RH503 (35-40 kDa) & EA & 1 & Paclitaxel & 5 & 121 & 47 & {$[25]$} \\
\hline PLGA50 : 50 (25kDa) & DCM & 2.5 & Paclitaxel & 5 & 318 & 89 & [26] \\
\hline Antibody-PLGA50: 50 (40 kDa) & Chloroform & 3 & Paclitaxel & 20 & 319 & 91 & [27] \\
\hline $\mathrm{R} 503 \mathrm{H}(0.32-0.44 \mathrm{dL} / \mathrm{g})$ & DCM & 5 & Pirfenidone & 50 & 225 & 31 & [28] \\
\hline PLGA50 : $50(40-100 \mathrm{kDa})$ & DCM & $1.25 \sim 5.0$ & Praziquantel & $10 \sim 30$ & $243 \sim 360$ & 82 & {$[29]$} \\
\hline mPEG-PLGA-PLL (n/a) & DCM-TFE & 2 & Rhodamine & 1 & 198 & 90 & {$[30]$} \\
\hline PGA-PLGA (103.4 kDa) & PC & 0.1 & Saquinavir & 10 & $120 \sim 300$ & 77 & {$[31]$} \\
\hline $\operatorname{PLGA}(\mathrm{n} / \mathrm{a})$ & $\mathrm{DCM}$ & 1 & $\mathrm{tPA}$ & 0.1 & $266 \sim 320$ & $66 \sim 71$ & {$[32]$} \\
\hline APRPG-PEG-PLA (n/a) & $\mathrm{DCM}$ & 1 & Fumagillin analog & 5 & 130 & 66 & {$[33]$} \\
\hline PLGA85 : $15(50-75 \mathrm{kDa})$ & $\mathrm{DCM}$ & 12.5 & Voriconazole & $8 \sim 100$ & $207 \sim 605$ & $8 \sim 63$ & [34] \\
\hline
\end{tabular}

Note: $\mathrm{n} / \mathrm{a}$, not available, not specified.

Polymer \%, polymer concentration in an organic solvent; D/P, the weight ratio of drug to polymer; EE, drug encapsulation efficiency; DCM, dichloromethane; DMSO, dimethylsulfoxide; EA, ethyl acetate; TFE, trifluoroethanol; PC, propyl carbonate; tPA, tissue-type plasminogen activator.

US 8445027 discloses the incorporation of bimatoprost, a prostamide derivative, into PLGA particles by $\mathrm{o}_{1} / \mathrm{o}_{2}$ emulsion methods [37]. Our literature survey shows that the size of PLGA nanoparticles prepared by an $\mathrm{o}_{1} / \mathrm{o}_{2}$ emulsion method is generally larger than that prepared by an $\mathrm{o} / \mathrm{w}$ emulsion method.

2.4. Oil-in-Water $(o / w)$ Salting-Out Technique. This technique involves the emulsification of a polymeric, drugcontaining organic solution into an electrolyte-saturated aqueous solution. A water-miscible organic solvent is used as a dispersed solvent for PLGA [38-42]. When a polymeric dispersed phase is mixed with an aqueous phase saturated with electrolytes, the salting-out effect prevents the mixing of the organic solvent with water, thereby producing a stable $\mathrm{o} / \mathrm{w}$ emulsion. A reverse salting-out effect is induced by adding an excessive amount of water to this emulsion. This triggers the diffusion of the water-miscible solvent out of emulsion droplets into the aqueous phase, which leads to formation of nanoparticles. Examples of electrolytes used in the salting-out process are magnesium acetate, magnesium chloride, calcium chloride, and sodium chloride. Benefits of this nanoencapsulation method include the use of nonhalogenated solvents to dissolve PLGA. In addition, this method does not require high-energy or high-pressure devices for emulsification. However, there is concern about the recycling of salts used in large quantities.
Following the salting-out principle, Konan et al. prepared PLGA nanoparticles [43]. Five milliliters of a PLGA solution (tetrahydrofuran or acetone) were homogenized in $20 \mathrm{~mL}$ of an aqueous PVA solution containing $60 \%$ of $\mathrm{MgCl}_{2}$, and $60 \mathrm{~mL}$ of water was added to this o/w emulsion. Optimization of processing conditions led to the formation of PLGA nanoparticles with a mean size below $200 \mathrm{~nm}$. The nanoparticles could be sterilized by filtration through a $0.22 \mu \mathrm{m}$ filter. An interesting work using the salting-out technique was also reported by Vettor et al. [44]. They encapsulated octinoxate, a UV filter, into PLGA nanoparticles by the following process: under vigorous stirring by a rotor/stator homogenizer, 3\% PVA aqueous solution containing $40 \% \mathrm{CaCl}_{2}$ was added into a dispersed phase made of acetone, PLGA, and octinoxate. Water was then added to the emulsion to convert emulsion droplets into PLGA nanoparticles. Under their experimental conditions, $d_{50 \%}$ was $670 \mathrm{~nm}$, and the drug encapsulation efficiency was $77.5 \%$. Nanoencapsulation of octinoxate into PLGA nanoparticles contributed to stabilizing the drug against photolysis up to $70.8 \%$. It was demonstrated that their PLGA nanoparticles in a gel dosage form preferentially stayed in the skin surface where the sunscreen agent was intended for [45].

2.5. Water-in-Oil-in-Water $\left(w_{1} / o / w_{2}\right)$ Emulsion Solvent Evaporation/Extraction. This method is used to encapsulate hydrophilic drugs into PLGA nanoparticles. A hydrophilic drug is dissolved in water $\left(\mathrm{w}_{1}\right)$, while PLGA is dissolved in 
TABLE 2: Summary of processing conditions of $\mathrm{w}_{1} / \mathrm{o} / \mathrm{w}_{2}$ emulsion methods and major characteristics of PLGA nanoparticles.

\begin{tabular}{|c|c|c|c|c|c|c|c|}
\hline Polymer (grade) & Solvent & Polymer \% & Drug & $\mathrm{D} / \mathrm{P}$ & Size $(\mathrm{nm})$ & $\mathrm{EE}(\%)$ & Reference \\
\hline Galactose-PLGA (n/a) & DCM-acetone & $\mathrm{n} / \mathrm{a}$ & Acyclovir & 10 & 198 & 61 & {$[46]$} \\
\hline $\begin{array}{l}\text { mPEG-PLGA-b-PLL } \\
(42.3 \mathrm{kDa})\end{array}$ & Chloroform & 1 & Adriamycin & 8 & 170 & 86 & {$[47]$} \\
\hline RG502H (n/a) & DCM & $\mathrm{n} / \mathrm{a}$ & Bevacizumab & $\mathrm{n} / \mathrm{a}$ & 197 & 82 & {$[48]$} \\
\hline PLGA50 : 50 (91.6-120 kDa) & Chloroform & 5 & BSA & $\mathrm{n} / \mathrm{a}$ & 290 & 65 & [49] \\
\hline RG503 (34 kDa) & DCM & 5 & BSA & 1 & 246 & 84 & {$[50]$} \\
\hline PLA-TPGS (25.3 kDa) & DCM & 1.5 & BSA & $6.7 \sim 40$ & $325 \sim 329$ & $53 \sim 80$ & [51] \\
\hline PLGA50 : 50 (0.4 dL/g) & DCM & 5 & BSA & 10 & 300 & 53 & {$[52]$} \\
\hline PLGA50 : 50 (30-60 kDa) & DCM & 1 & CTP (52 kDa) & $\mathrm{n} / \mathrm{a}$ & 272 & 60 & {$[53]$} \\
\hline $\operatorname{PLGA}(\mathrm{n} / \mathrm{a})$ & DCM-acetone & 1.25 & CAA & 0.8 & 187 & 76 & {$[54]$} \\
\hline PLGA50 : 50 (1,234 kDa) & Chloroform & 3 & pDNA (7.3 kbp) & $0.55 \sim 1.1$ & $538 \sim 644$ & $9 \sim 32$ & {$[55]$} \\
\hline PLGA50: 50 (40-75 kDa) & DCM & $0.1 \sim 5$ & Doxorubicin & $2 \sim 100$ & 100 & $35 \sim 85$ & {$[56]$} \\
\hline PLGA50 : 50-chitosan (n/a) & DCM & $\mathrm{n} / \mathrm{a}$ & Exendin-4 & $\mathrm{n} / \mathrm{a}$ & $275 \sim 310$ & $\mathrm{n} / \mathrm{a}$ & {$[57]$} \\
\hline $\begin{array}{l}\text { PLGA50 : 50-chitosan } \\
(15 \mathrm{kDa})\end{array}$ & $\mathrm{DCM}$ & 1.67 & 5-Fluorouracil & 10 & $198 \sim 280$ & $23 \sim 29$ & {$[58]$} \\
\hline $\mathrm{R} 502 \mathrm{H}(12 \mathrm{kDa})$ & DCM & 5 & Gentamicin & 3.5 & $241 \sim 252$ & $6 \sim 22$ & {$[59]$} \\
\hline RG502H (13.8 kDa) & EA & $\mathrm{n} / \mathrm{a}$ & Gentamicin & $1 \sim 5$ & 320 & $13 \sim 47$ & {$[60]$} \\
\hline PLGA75 : $25(20 \mathrm{kDa})$ & DCM & $3,6,20$ & GFP siRNA & $0.24,0.6$ & $224 \sim 428$ & $11 \sim 58$ & {$[61]$} \\
\hline RG503 (34 kDa) & DCM & 5 & Insulin & 5 & 276 & 68 & {$[50]$} \\
\hline RG503H (n/a) & DCM & 1.5 & hgp100, TRP2 & 1 & 80 & $8 \sim 40$ & {$[62]$} \\
\hline PLGA50 : $50(30 \mathrm{kDa})$ & DCM & 3.3 & LA & $0.2,3.85$ & 300 & $49 \sim 72$ & {$[63]$} \\
\hline RG502H (10-12 kDa) & $\mathrm{DCM}$ & 3 & Ovalbumin & 1.67 & 182 & 54 & {$[64]$} \\
\hline PLGA50 : 50 (7-17 kDa) & $\mathrm{DCM}$ & 2.9 & Ovalbumin & 20 & 358 & 36 & {$[65]$} \\
\hline PLGA50 : 50 (40-75 kDa) & DCM & 2 & PEI-rhodamine B & 0.5 & $117 \sim 130$ & $45 \sim 75$ & {$[66]$} \\
\hline PLGA50 : 50 (n/a) & $\mathrm{DCM}$ & $\mathrm{n} / \mathrm{a}$ & pRedN-1 DNA & $\mathrm{n} / \mathrm{a}$ & 998 & 10 & {$[67]$} \\
\hline PLGA50 : 5 (45-75 kDa) & $\mathrm{DCM}$ & $1 \sim 5$ & TIMP-1 (28 kDa) & $0.2 \sim 1.0$ & $81 \sim 433$ & $62 \sim 80$ & {$[68]$} \\
\hline
\end{tabular}

Note: n/a, not available, not specified.

All abbreviations are the same as those described in Table 1; BSA, bovine serum albumin; CTP, Chlamydia trachomatis protein; CAA, collagen adhesin antigen; LA, Leishmania antigen; TIMP-1, tissue inhibitor of matrix metalloproteinase-1.

a water-immiscible solvent. The aqueous phase $\left(\mathrm{w}_{1}\right)$ is emulsified in the polymeric organic solution. This primary $\mathrm{w}_{1} / \mathrm{o}$ emulsion is mixed again in an emulsifier-containing aqueous phase $\left(\mathrm{w}_{2}\right)$. This results in the formation of a $\mathrm{w}_{1} / \mathrm{o} / \mathrm{w}_{2}$ double emulsion. The organic solvent is removed to produce PLGA nanoparticles. In most cases, this method yields poor encapsulation efficiencies toward small-molecular-weight drugs, because of their escaping tendency into the $\mathrm{w}_{2}$ phase during nanoencapsulation. Table 2 summarizes the experimental conditions of $\mathrm{w}_{1} / \mathrm{o} / \mathrm{w}_{2}$ emulsion-based nanoencapsulation methods and the major nanoparticle quality.

2.6. Water-in-Oil-in-Oil $\left(w / o_{1} / o_{2}\right)$ Emulsion Solvent Evaporation/Extraction. The $\mathrm{w}_{2}$ phase of a $\mathrm{w}_{1} / \mathrm{o} / \mathrm{w}_{2}$ emulsion-based method is replaced with an organic solvent that is miscible with the dispersed organic solvent but is antisolvent for PLGA and drug. For example, Afshari et al. emulsified an aqueous methotrexate solution in methylene chloride where PLGA and Span 80 were dissolved [69]. This w/o $\mathrm{o}_{1}$ emulsion was added into liquid paraffin $\left(\mathrm{o}_{2}\right)$ under homogenization. After stirring the $\mathrm{w} / \mathrm{o}_{1} / \mathrm{o}_{2}$ emulsion for $5 \mathrm{hr}$, PLGA nanoparticles were collected by centrifugation and washed with $n$-hexane.
Moderate encapsulation efficiency of $51.2 \%$ was attained with this nanoencapsulation method.

\section{Nanoprecipitation}

When oil dissolved in a water-miscible organic solvent is mixed with a large amount of water, spontaneous emulsification takes place to make a kinetically stable nanoemulsion. This so-called Ouzo effect is a process by which hydrophobic solutes are dispersed in water as nanosized droplets or particles [70]. When the Ouzo emulsification process is applied for preparing polymeric nanoparticles, the nanoencapsulation method is referred to as nanoprecipitation. In this practice, a hydrophobic polymer material is first dissolved in a watermiscible solvent. This polymeric dispersed phase is mixed with an aqueous phase by use of a low-energy mixing device. The solution resulting from the mixing of the polymeric phase with antisolvent (i.e., water) is a nonsolvent for the polymer solutes. As a consequence, hydrophobic polymer solutes become aggregated to form nanosized particles. When Fessi et al. used this nanoprecipitation technique to prepare PLGA nanoparticles, they named it as the solvent displacement 
TABLE 3: Summary of processing conditions of nanoprecipitation and major characteristics of PLGA nanoparticles.

\begin{tabular}{|c|c|c|c|c|c|c|c|}
\hline Polymer (grade) & Solvent & Polymer (\%) & Drug (D) & $\mathrm{D} / \mathrm{P}$ & Size $(\mathrm{nm})$ & $\mathrm{EE}(\%)$ & Reference \\
\hline PLGA50 : 50 (0.2 dL/g) & Acetone & 2.5 & Anastrazole & 5 & $118 \sim 180$ & $37 \sim 62$ & {$[74]$} \\
\hline RGP d series (n/a) & Acetone & 4 & Atorvastatin & 12 & $76 \sim 172$ & $3 \sim 51$ & {$[75]$} \\
\hline PLGA50 : 50 (45-75 kDa) & Acetone-ethanol & 2 & Azelaic acid & 50 & 252 & 72 & {$[76]$} \\
\hline RGP d 50155 & PEG 400 & $0.3 \sim 1.3$ & BSA, lysozyme & $12.5 \sim 50$ & $95 \sim 394$ & Up to 35 & {$[77]$} \\
\hline PLGA (n/a) & Acetone & 1.7 & Chelidonine & 20 & 123 & 83 & {$[78]$} \\
\hline PLA (42 kDa) & Acetone-ethanol & 0.6 & Chloroaluminium & 3.33 & 115 & 57 & {$[79]$} \\
\hline PLGA75 : $25(10 \mathrm{kDa})$ & Acetone & 1 & Coenzyme-Q & 10 & 100 & 88 & {$[80]$} \\
\hline PLGA50:50 (7-17 kDa) & Tetrahydrofuran & 0.2 & Curcumin & 100 & 79 & 92 & [81] \\
\hline PLGA (n/a) & Acetone & 1.25 & Docetaxel & 10 & 139 & 75 & {$[82]$} \\
\hline RG502H (n/a) & Acetone & 0.375 & Docetaxel & $0.1,1$ & $157 \sim 172$ & $17 \sim 23$ & {$[83]$} \\
\hline PLA-TPGS (25.3 kDa) & Acetone-ethanol & 1.01 & Docetaxel & $\mathrm{n} / \mathrm{a}$ & 122 & 80 & {$[84]$} \\
\hline RG502 (12 kDa) & Acetone & 1.5 & Cannabinoid & $6 \sim 20$ & $291 \sim 315$ & $71 \sim 90$ & {$[85]$} \\
\hline PLGA75 : 25 (75-120 kDa) & PC & 2 & Estradiol benzoate & 1.5 & ca. $70 \sim 250$ & 67 & {$[86]$} \\
\hline PLGA-PEG (34 kDa) & Acetone & 0.36 & Flurbiprofen & 1.67 & 123 & 81 & [87] \\
\hline PLGA50 : 50 (40-75 kDa) & Acetone-ethanol & 0.88 & Loperamide & 50 & 174 & 41 & {$[88]$} \\
\hline RG 502H (n/a) & Acetone & 0.375 & Melatonin & $1,3,5$ & ca. 100 & $6 \sim 44$ & [89] \\
\hline PLGA (n/a) & Acetone & 1.67 & Coumarin & 20 & 117 & 88 & {$[90]$} \\
\hline PLGA50: $50(40.1 \mathrm{kDa})$ & DMA & 2 & Norfloxacin & 100 & 323 & 15 & [91] \\
\hline PLGA (n/a) & Acetone & 1 & Paclitaxel & 2 & 87 & 2 & {$[92]$} \\
\hline RG502H (n/a) & Acetonitrile & 2.5 & Paclitaxel & 4 & $<1,000$ & 86 & [93] \\
\hline PLGA50 : 50 (n/a) & Acetone & 2 & Paclitaxel & $1 \sim 10$ & $250 \sim 300$ & $5 \sim 91$ & {$[94]$} \\
\hline PLGA (60 kDa) & Acetone & 0.24 & Ropivacaine & $0.25,0.5$ & 163 & 4 & {$[95]$} \\
\hline PLGA50 : 50 (41 kDa) & Acetone & 1.25 & Testosterone & 40 & 202 & 27 & {$[96]$} \\
\hline PLGA-PEG (n/a) & Acetonitrile & 1 & Thymoquinone & 5 & $150 \sim 200$ & 3 or 94 & [97] \\
\hline RG502H (12 kDa) & Acetone & 2 & Tramadol & 10 & 141 & 74 & {$[98]$} \\
\hline PLGA50 : 50 (50-75 kDa) & Acetone & 0.5 & Xanthone & 1.5 & 96 & 43 & [99] \\
\hline
\end{tabular}

Note: $\mathrm{n} / \mathrm{a}$, not available, not specified.

All abbreviations are the same as those described in Table 1; DMA, dimethylacetamide.

method [71]. Some researchers name nanoprecipitation as a solvent-dialysis method, in case when solvent removal is performed by dialysis [72]. The solvent should be water-miscible and be easily removed by evaporation, extraction, and/or combination of both [11, 73]. Common solvents for nanoprecipitation are acetone, acetonitrile, dimethylacetamide, dimethylformamide, dimethylsulfoxide, 2-pyrrolidone, $\mathrm{N}$ methyl-2-pyrrolidone, polyethylene glycol, and tetrahydrofuran. Among them, acetone is one of the most preferred solvents of choice. Sometimes, a binary mixture of solvents (e.g., acetone-ethanol) is used with this method.

Table 3 summarizes representative experimental conditions and the characteristics of PLGA nanoparticles prepared by nanoprecipitation. The size of PLGA nanoparticles and drug encapsulation efficiency are influenced by various factors: solvent, PLGA, drug : PLGA ratio, surfactant, polymeric phase: antisolvent phase volume ratio, and the like. It should also be emphasized that the mode of mixing a polymeric dispersed phase with antisolvent influences the quality of PLGA nanoparticles. The reason is that solvent shifting/displacement may proceed under a nonuniform environment. If put otherwise, depending upon the mode of mixing, the situation at which initial mixing occurs can be different from that at a final stage. Mixing can be performed in various ways: (i) the one-shot pouring of antisolvent (water) into a PLGA organic solution; (ii) controlled, drop-wise addition of water to a PLGA organic solution; (iii) a singleshot pouring of a PLGA organic solution onto water; (iv) controlled, drop-wise addition of a PLGA organic solution into water; and ( $\mathrm{v}$ ) dialysis of a polymeric dispersed phase using water. Also, the processing conditions can be further diversified by predissolving an organic solvent in water before mixing.

Nanoprecipitation does not need the use of high-energy/ high-shear/high-pressure mixing devices, because the formation of PLGA nanoparticles is driven by spontaneous diffusion of an organic solvent into water. Yet, PLGA nanoparticles produced by nanoprecipitation are smaller than those prepared by emulsion solvent evaporation/extraction techniques. Another merit of nanoprecipitation is that nonhalogenated organic solvents are used as dispersed solvents for PLGA. They are less toxic and more eco-friendly than halogenated organic solvents used in emulsion solvent evaporation/extraction processes. A major drawback is that the success of preparing nanoparticles is restricted only to a narrow region of the polymer/solvent/antisolvent composition map, 
the so-called Ouzo region $[70,100]$. Beyond the Ouzo region, microparticles rather than nanoparticles are produced. It should also be noted that, during nanoprecipitation, solvent shift happens as a result of the dilution of PLGA solutes in antisolvent (water). This causes the nucleation of very small aggregates of PLGA molecules, aggregation of these nuclei, and formation of nanoparticles. Therefore, a large amount of water should be added into a PLGA dispersed phase. A series of these events must take place at low polymer concentrations. Otherwise, the solvent-swollen particles are prone to coalescence, thereby generating micron-sized particles. If an organic solvent is not a theta solvent for PLGA, nanoprecipitation causes the formation of large polymeric aggregates. Finally, the incomplete removal of residual solvents from nanoparticles brings about severe aggregation of PLGA nanoparticles during ultracentrifugation and/or freeze-drying.

\section{Spontaneous Emulsion Solvent Diffusion (SESD) Technique}

Strictly speaking, nanoprecipitation is a single-step formation of polymeric dispersion in water. In the field of the preparation of PLGA nanoparticles, this method has been slightly modified to include the steps of emulsion formation and nanoparticle hardening. Based on this strategy, Niwa et al. proposed the so-called SESD technique [101]. Its key strategy was to use a binary solvent mixture consisting of water-immiscible and water-miscible solvents (e.g., methylene chloride-acetone). Ibrahim et al. also used a methylene chloride-acetone binary system to prepare celecoxib-loaded PLGA nanoparticles [18]. Acetone, which is miscible with methylene chloride and water, helps decrease the interfacial tension between methylene chloride and water. Additionally, the interfacial turbulence caused by the rapid diffusion of acetone from emulsion droplets to water contributes to the spontaneous breakups of emulsion droplets into nanosized ones. Solvent evaporation follows to remove methylene chloride from the nanoemulsion, so as to produce solid PLGA nanoparticles. These are the underlying principles of the SESD technique. On the basis of these considerations, SESD can be regarded as a hybrid of emulsion solvent evaporation/extraction and nanoprecipitation. There are other reports that exploit the importance of a solvent system for manipulating the physicochemical attributes of resultant nanoparticles $[102,103]$.

\section{Membrane Emulsification Technique}

Nakashima et al. proposed the Shirasu Porous Glass (SPG) membrane-emulsification technique to prepare various kinds of particulate matters $[104,105]$. Since then, this technique has been widely explored for the preparation of liposomes, microparticles, and nanoemulsions [106-110]. Briefly, this emulsification system forces a polymeric dispersed phase to permeate through a porous membrane into an aqueous continuous phase. The resultant emulsion is subject to the process of solvent removal, thereby generating nanoparticles.
The membrane properties (pore size, spatial distribution, and tortuosity) play a decisive role on the size of the emulsion droplets and/or nanoparticles. This feature is distinctive from other emulsification systems using high-energy sonicators, high-shear rotor/stator homogenizers, or high-pressure microfluidizers. While these conventional mechanical methods require high energy input $\left(10^{6}-10^{8} \mathrm{~J} \cdot \mathrm{m}^{-3}\right)$, the membrane emulsification method uses relatively low energy input $\left(10^{4}-\right.$ $\left.10^{6} \mathrm{~J} \cdot \mathrm{m}^{-3}\right)$.

For membrane emulsification, either a polymeric dispersed phase or a premix (i.e., a ready-made coarse emulsion) is passed through a membrane. For example, Wei et al. emulsified a PLGA dispersed phase in an aqueous phase by using a low-speed rotor/stator homogenizer [111]. This coarse emulsion had large droplet sizes with a broad size distribution. By extruding the premix through a SPG membrane $(1.2 \sim 9 \mu \mathrm{m}$ pore size) by use of nitrogen gas pressure, they were able to produce uniform PLGA nanoparticles. Yu et al. also encapsulated docetaxel into PLGA-TPGS nanoparticles by combining preemulsification and SPG membraneemulsification [112]. The docetaxel/PLGA-TPGS/ethyl acetate dispersed phase was mixed in an aqueous phase by a magnetic stirrer. The resulting premix was poured into the SPG membrane apparatus. Nitrogen gas pressure was applied to let the premix pass through the membrane. Solvent removal led to the formation of hardened nanoparticles. The process parameters that they investigated were the type and concentration of a stabilizer (PVA, TPGS, and Pluronic F68), the volumetric ratio of dispersed phase to aqueous phase $(1: 10 \sim$ $1: 20)$, transmembrane cycle (1 5), operation pressure (0.6 $1.2 \mathrm{MPa})$, and membrane pore size $(0.5 \sim 1.5 \mu \mathrm{m})$. Depending upon their process conditions, the mean diameter of their nanoparticles ranged from 236 to $462 \mathrm{~nm}$ (Figure 1). Smaller nanoparticles appeared with smaller membrane pore sizes, and docetaxel encapsulation efficiency varied from 18.9 to $85.7 \%$. At present, the membrane emulsification technique is somewhat limited to the preparation PLGA nanoparticles greater than $200 \mathrm{~nm}$.

\section{Hydrophobic Ion Pairing- (HIP-) and Reverse Micelle-Hybridized o/w Emulsion Technique}

A number of attempts have been made to increase the loading efficiency of hydrophilic drugs into PLGA nanoparticles. One relevant strategy is to enhance the liposolubility of watersoluble drugs by use of a hydrophobic ion pairing (HIP) technique [113]. Briefly, bis(2-ethylhexyl) sulfosuccinate sodium salt (AOT) was dissolved in methylene chloride, which was then vigorously stirred in an aqueous gentamicin sulfate solution. This caused a complexation reaction between the anionic AOT and the cationic gentamicin. The hydrophobic ionic complex, after being recovered from the organic phase by evaporation, was used for an o/w emulsion nanoencapsulation process. Depending upon the type of PLGA and the theoretical payload of gentamicin-AOT, the nanoparticles had mean diameters ranging from 252 to $330 \mathrm{~nm}$, with encapsulation efficiencies of almost $100 \%$. In a following 


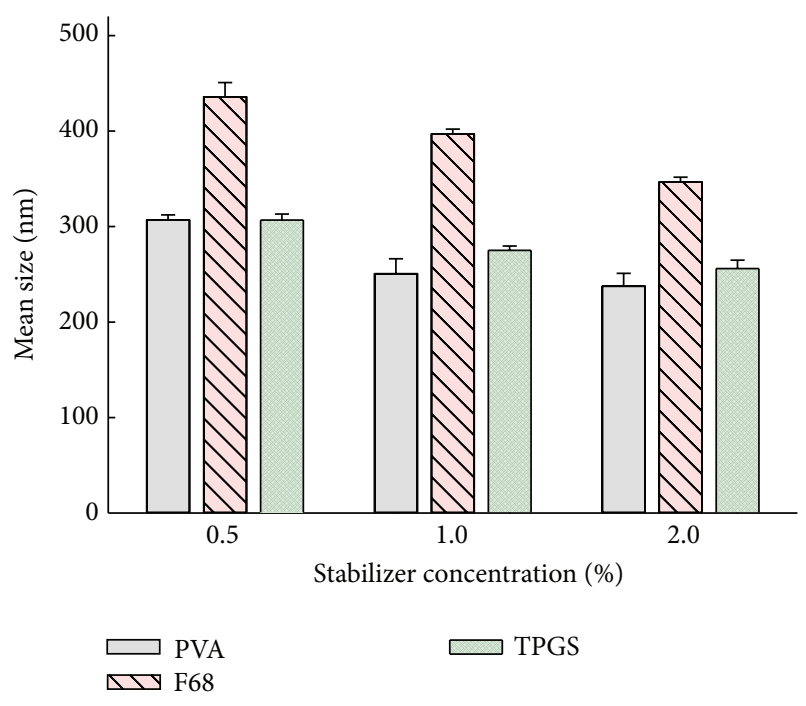

(a)

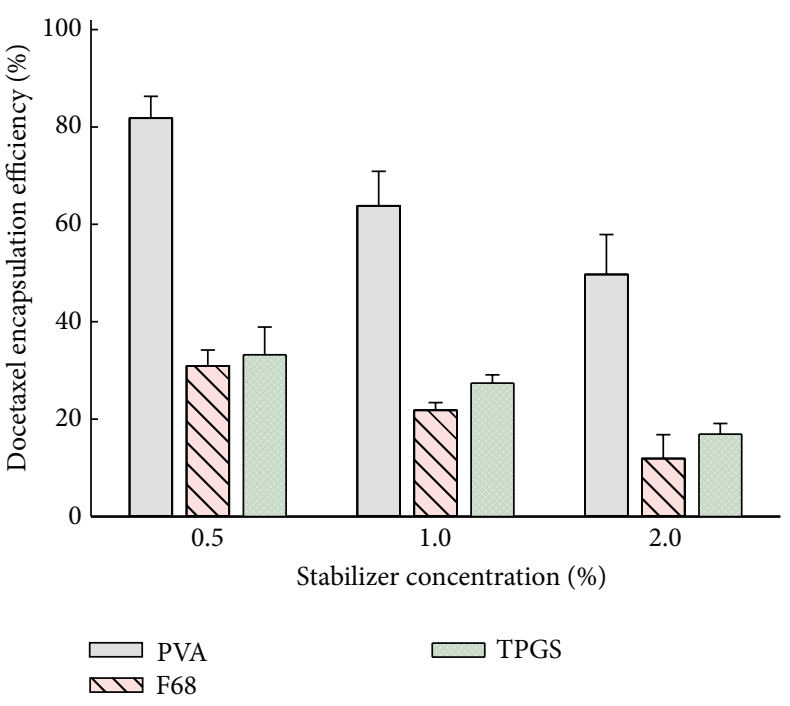

(b)

FIGURE 1: Effects of the type and concentration of a stabilizer upon (a) nanoparticle size and (b) docetaxel encapsulation efficiency \% (Yu et al., [112], publisher and licensee Dove Medical Press Ltd.).

animal study, Imbuluzqueta et al. demonstrated that these PLGA nanoparticles had no apparent toxic effects upon the liver and the spleen [114]. It was anticipated that they might be applicable for the treatment of brucellosis and tuberculosis.

Similar strategies utilizing the HIP principle have been applied for the encapsulation of water-soluble compounds and biomacromolecules [115-117]. For example, Yang et al. complexed a water-soluble peptide (melittin) with sodium dodecyl sulfate in an aqueous solution [117]. Their electrostatic interaction led to the formation of white precipitates, which were recovered by centrifugation and freeze-dried. When this complex was used for nanoencapsulation, the drug encapsulation efficiency amounted to $99.6 \%$. Lipids are also used in replacement of surfactants for HIP approaches, as shown in the work of Cui et al. [118]. They prepared a hydrophobic complex of insulin-phosphatidylcholine by an anhydrous cosolvent lyophilization method. Their PLGA nanoparticles prepared following an o/w emulsion process had a median diameter of $345 \mathrm{~nm}$ with the insulin encapsulation efficiency of $90.4 \%$.

All HIP-hybridized o/w nanoencapsulation techniques described above proceed with dissolving hydrophobic ion complexes in a dispersed organic solvent. Instead, Kim et al. proposed a reverse micelle-based o/w emulsion solvent evaporation/extraction method [119]. In their work, oxytetracycline $\mathrm{HCl}$ was directly dissolved in the reverse micelles consisting of cetyltrimethylammonium bromide, water, and ethyl formate. PLGA was then dissolved in the reverse micellar solution, and this dispersed phase was emulsified in an aqueous solution. A solvent extraction step was carried out to harden emulsion droplets into PLGA microspheres. Under their optimized processing conditions, the encapsulation efficiency of oxytetracycline $\mathrm{HCl}$ was $24.9 \%$. The amount of water used to prepare reverse micelles was found to be one of key parameters affecting the drug encapsulation efficiency.

\section{Microfluidic Technology Platforms}

Microfluidics refers to the science of dealing with fluids in microchannels that have at least one dimension smaller than $1,000 \mu \mathrm{m}$ [120]. Microfluidic devices are capable of achieving rapid mass transfer and efficient mixing [121]. A liquid flow pattern is controlled by microchannel geometry and flow rate. Syringe pumps are usually used to trigger pressuredriven flow of liquids through microchannels. Most studies making use of microfluidic platforms were primarily focused on the preparation of emulsion droplets and polymeric microparticles, but more attention is being given to the generation of nanoemulsions and polymeric nanoparticles nowadays [122-125].

Microfluidic methods for generating emulsion droplets can be classified into three categories: (i) breakup in coflowing streams; (ii) breakup in cross-flowing streams in a Tshaped junction; and (iii) breakup in elongational flow in a flow focusing geometry [126-128]. The first mode of breakup is rarely applied for the preparation of PLGA nanoparticles. Most reports deal with the adaptation of microfluidic Tjunction and flow focusing geometries for the production of PLGA nanoparticles. Since the mixing of two or more liquids in a microfluidic device is done rapidly, a microfluidic nanoencapsulation platform works well with nanoprecipitation.

A microfluidic device utilizing T-junction was first proposed by Thorsen et al. in 2001 [129]. Since then, many studies have used microfluidic T-junction geometries for various applications. Xie and Smith used this device to prepare PLGA nanoparticles (Figure 2(a)) [130]. They inserted a syringe needle (an inner diameter $110 \mu \mathrm{m}$ ) into a Tygon tube (inner diameter $2.4 \mathrm{~mm}$, outer diameter $4 \mathrm{~mm}$ ) through which water stream flowed. By feeding a PLGA-dissolved acetonitrile solution into the syringe needle, they produced 


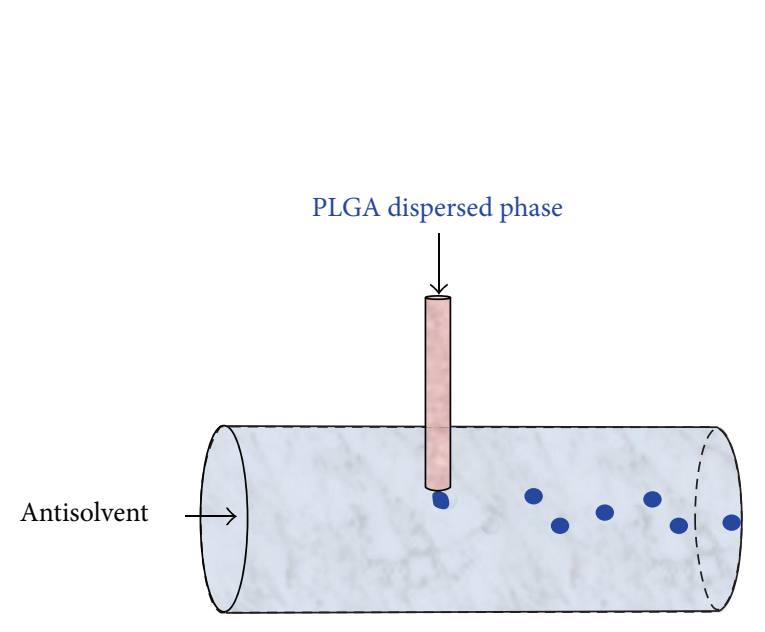

(a)

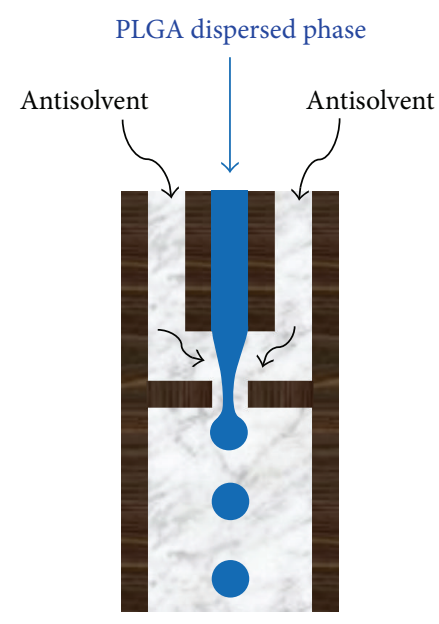

(b)

Figure 2: (a) Preparation of PLGA nanoparticles by use of a microfluidic T-junction device. (b) Mixing a PLGA dispersed phase with antisolvent in a microfluidic device with a flow focusing geometry.

PLGA nanoparticles in the range of 140 to $500 \mathrm{~nm}$. These nanoparticles had narrower size distributions than those prepared by a conventional, bulk scale nanoprecipitation process (Figure 3). In order to differentiate their microfluidic nanoprecipitation process from the traditional nanoprecipitation process, they coined the new term of Fluidic NanoPrecipitation System (FNPS). This is also referred to as flash nanoprecipitation [131].

A microfluidic T-junction device can be modified to coat specific macromolecules on polymeric nanoparticles, or to prepare multiphase particles $[93,132]$. For example, Xie et al. developed a microfluidic system with dual inlet channels perpendicularly inserted into a tube [93]. Compared to the previous microfluidic T-junction device (Figure 2(a)) with one inlet channel, the two inlet channels in this device allow the feeding of two types of dispersed phases into the tube through which water stream flows. In their work, one dispersed phase was made of PLGA, paclitaxel, and acetone. The other dispersed phase was prepared by emulsifying an aqueous doxorubicin $\mathrm{HCl}$ in PLGA-dissolved methylene chloride. Under these experimental conditions, they were able to produce Janus nanoparticles encapsulating hydrophobic paclitaxel on one side and hydrophilic doxorubicin $\mathrm{HCl}$ on the other side. Impressively, encapsulation efficiencies of both drugs were $\geq 80 \%$.

Anna et al. and Dreyfus et al. demonstrated the mixing of two immiscible fluids by microfluidic flow focusing $[133,134]$. In the field of microencapsulation, flow focusing is usually utilized for loading drugs into polymeric microparticles [135137]. Flow focusing can be summarized by the following steps: (i) an aqueous continuous phase (often referred to as the focusing fluid) is introduced from two side channels, while a polymeric dispersed phase (referred to as the focused fluid) is injected into the central channel (Figure 2(b)); (ii) the two liquid streams are forced by syringe pumps to pass through a narrow orifice of a flow focusing atomizer/nozzle; (iii) the focused fluid is squeezed by the focusing fluid flow; (iv) both phases pass through the small orifice that is located downstream of the three channels; and (v) the removal of the dispersed organic solvent in a collection chamber leads to the generation of PLGA nanoparticles.

Recently, Martín-Banderas et al. has utilized the flow focusing technique to encapsulate a water-soluble compound, gemcitabine, into PLGA nanoparticles [138]. Surprisingly, the gemcitabine encapsulation efficiency was $97.3 \%$, a significantly higher value than that of $57.5 \%$ observed with a typical $\mathrm{w}_{1} / \mathrm{o} / \mathrm{w}_{2}$ emulsion technique. The quick one-step formation of gemcitabine-loaded nanoparticles might be held accountable for the better drug encapsulation efficiency attained with flow focusing. In the work of Karnik et al., a dispersed phase consisting of PLGA-PEG and acetonitrile was focused by two intersecting flow streams of water [139]. An excellent discussion was provided in relation to the mechanisms of selfassembly of PLGA-PEG nanoparticles during flow focusing, as well as the dependence of nanoparticle size upon mixing conditions (slow mixing versus rapid mixing). Also, Lim et al. have reported a 3D multiparallel microfluidic system not only to prevent the fouling of microchannels by PLGA but also to increase the scale of nanoparticle production [140]. They were able to tune the size of PLGA-PEI nanoparticles from 13 to $150 \mathrm{~nm}$ simply by changing the flow ratio of the dispersed phase to antisolvent.

During conventional nanoencapsulation processes, the initial mixing of a polymeric dispersed phase in an aqueous phase takes place at a bulk, large scale. This bulk process does not have the capability of directing the process of PLGA precipitation in a controlled way. By comparison, microfluidic devices allow more precise controls over mixing and solvent removal processes. In addition, microfluidic approaches provide higher encapsulation efficiencies for hydrophilic drugs than bulk nanoencapsulation processes do. Particular features and major limitations of various preparation methods of PLGA nanoparticles discussed so far are summarized in Table 4. 
TABLE 4: Comparison of various emulsion-templated preparation methods of PLGA nanoparticles.

\begin{tabular}{|c|c|c|}
\hline Nanoencapsulation & Particular features & Major issues and limitation \\
\hline w/o phase separation & $\begin{array}{l}\text { High EE\% for water-soluble drugs; a phase } \\
\text { inducer is used for nanoparticle hardening }\end{array}$ & $\begin{array}{l}\text { Handling and disposal of oil; difficulty in } \\
\text { controlling coacervation; relatively large-sized } \\
\text { nanoparticles }\end{array}$ \\
\hline $\mathrm{w}_{1} / \mathrm{o} / \mathrm{w}_{2}$ emulsion & $\begin{array}{l}\text { A well-established process for water-soluble } \\
\text { drugs }\end{array}$ & $\begin{array}{l}\text { Poor drug EE\%; strategies are needed for } \\
\text { improving drug EE\%; limited drug payload; } \\
\text { drug instablization during emulsification }\end{array}$ \\
\hline HIP technique & High EE\% for water-soluble drugs & $\begin{array}{l}\text { Toxic residual ionic surfactants in } \\
\text { nanoparticles; alternatives to ionic surfactants } \\
\text { are recommended for complexation }\end{array}$ \\
\hline $\begin{array}{l}\mathrm{o} / \mathrm{w} \text { solvent } \\
\text { evaporation }\end{array}$ & $\begin{array}{l}\text { A platform easily adapted for hydrophobic } \\
\text { drugs }\end{array}$ & $\begin{array}{l}\text { Use of volatile halogenated organic solvents; } \\
\text { solvent toxicity; residual solvent content }\end{array}$ \\
\hline o/w solvent extraction & $\begin{array}{l}\text { A well-established technique for hydrophobic } \\
\text { drugs; a nonhalogenated solvent is used }\end{array}$ & $\begin{array}{l}\text { Generation of large quantities of waste-stream; } \\
\text { incomplete solvent removal; nanoparticle } \\
\text { aggregation }\end{array}$ \\
\hline $\mathrm{o}_{1} / \mathrm{o}_{2}$ emulsion & $\begin{array}{l}\text { A preparation method for water-insoluble } \\
\text { drugs; processing solvents are nonhalogenated }\end{array}$ & $\begin{array}{l}\text { Disposal and/or recycling of waste oil; poor } \\
\text { EE\% caused by drug partition to } \mathrm{O}_{2}\end{array}$ \\
\hline $\mathrm{o} / \mathrm{w}$ salting-out & $\begin{array}{l}\text { Moderate EE\% for hydrophobic drugs; } \\
\text { water-miscible, nonhalogenated solvent; } \\
\text { low-energy mixing device }\end{array}$ & $\begin{array}{l}\text { Use of large quantities of salting-out agents } \\
\text { (e.g., salts/electrolytes); recycling of } \\
\text { salts/electrolytes }\end{array}$ \\
\hline Nanoprecipitation & $\begin{array}{l}\text { A water-miscible, nonhalogenated solvent; } \\
\text { a single-step process for loading hydrophobic } \\
\text { drugs; low-energy mixing device; smaller } \\
\text { nanoparticle size }\end{array}$ & $\begin{array}{l}\text { A narrow Ouzo region for nanoparticle } \\
\text { formation; a polymeric concentration of } \\
\text { a dispersed phase is low; nanoparticle } \\
\text { aggregation due to incomplete solvent removal }\end{array}$ \\
\hline SESD & $\begin{array}{l}\text { Modified nanoprecipitation for hydrophobic } \\
\text { drugs }\end{array}$ & $\begin{array}{l}\text { A binary solvent mixture containing } \\
\text { a halogenated one }\end{array}$ \\
\hline $\begin{array}{l}\text { Membrane } \\
\text { emulsification }\end{array}$ & $\begin{array}{l}\text { Low-energy mixing device; homogeneous } \\
\text { nanoparticle population }\end{array}$ & $\begin{array}{l}\text { Membrane properties decide nanoparticle } \\
\text { quality; relatively bigger nanoparticle sizes } \\
\text { (e.g., } \geq 200 \mathrm{~nm} \text { ) }\end{array}$ \\
\hline Microfluidic technique & $\begin{array}{l}\text { Precise control over nanoparticle formation; } \\
\text { high drug EE\% for various types of drugs; } \\
\text { multiple drug loads; low-energy mixing device }\end{array}$ & $\begin{array}{l}\text { Scalability; limitation in commercial scale } \\
\text { production }\end{array}$ \\
\hline
\end{tabular}

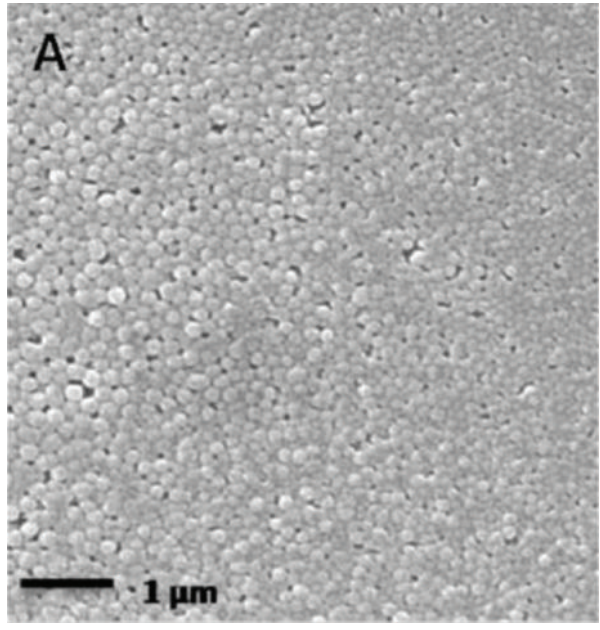

(a)

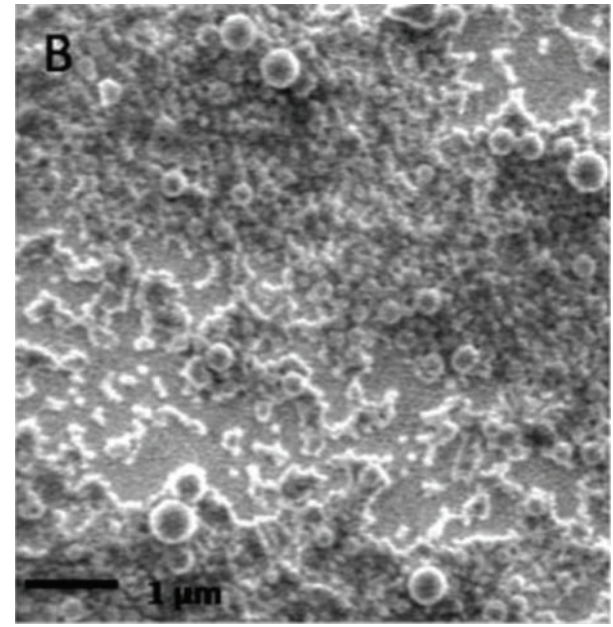

(b)

FIGURE 3: SEM micrographs of PLGA nanoparticles prepared by (a) the microfluidic approach and (b) the conventional nanoprecipitation method using bulk solutions. The mean diameters of PLGA nanoparticles prepared by (a) and (b) methods are $148 \pm 14 \mathrm{~nm}$ and $211 \pm 70 \mathrm{~nm}$, respectively (Xie and Smith, [130], publisher and licensee BioMed Central Ltd.). 


\section{Improvement of Drug Encapsulation Efficiency}

Drug encapsulation efficiency is affected by numerous factors including: PLGA property, solvent characteristics, drugPLGA interaction, drug miscibility in PLGA, drug solubility in an organic solvent and its antisolvent, drug stability, and manufacturing methods. In particular, fragile biomacromolecules (e.g., siRNA, DNA, and proteins) are prone to destabilization, aggregation, and/or degradation during nanoencapsulation. A series of these events happen because they are exposed to organic solvent, organic solvent/water interface, hydrophobic PLGA matrix, heat, shear force, cavitation, or free radicals [141]. Protein instability inside PLGA particles is also an important issue [142, 143]. Ibrahim et al. reported that insulin encapsulated in PLGA particles underwent chemical degradation reactions such as deamidation [144]. The ester backbone in PLGA is prone to autocatalytic degradation due to free carboxylic end groups. This problem can be solved by coencapsulating drugs with bases (e.g., magnesium hydroxide, zinc carbonate) into PLGA particles [145]. The coencapsulated bases neutralize the acidic environment of degrading PLGA matrices, thereby contributing to maintaining drug stability. Another problem is that drug molecules tend to diffuse out of nanoemulsions or embryonic nanoparticles when they undergo stirring to complete the process of nanoparticle hardening. There are a number of strategies developed to improve drug encapsulation efficiency, and some representative strategies are briefly discussed below.

8.1. Modulation of the Surface Property of PLGA. The type of PLGA can affect drug stability, encapsulation efficiency, release kinetics, and in vivo behavior. For example, PEGylation of PLGA provides nanoparticles several merits in terms of stabilization of emulsion droplets and protein encapsulation efficiency. During $\mathrm{w}_{1} / \mathrm{o} / \mathrm{w}_{2}$ nanoencapsulation, PEG chains are present toward the $\mathrm{w}_{1}$ phase containing a protein. This orientation keeps protein molecules off the water/organic solvent interface, thereby minimizing the interface-induced denaturation and aggregation [146]. Surface modification of PLGA nanoparticles with a cell penetrating polymer, poly(guanidinium oxanorbornene), resulted in an improved belinostat loading from $39 \pm 0.63$ to 129 $\pm 3.1 \mu \mathrm{g} / \mathrm{mg}$ of nanoparticles [147]. Nahar and Jain also reported that the drug content in polymeric nanoparticles was influenced by the type of PLGA [14]. Using plain PLGA and mannose-PEG-PLGA as nanoparticle-forming materials, they prepared amphotericin B-containing nanoparticles via an $\mathrm{o} / \mathrm{w}$ emulsion method. Encapsulation efficiencies of amphotericin B observed with plain PLGA nanoparticles and mannose-PEG-PLGA ones were $53.0 \pm 1.5$ and $81.2 \pm$ $2.1 \%$, respectively. Better miscibility and interactions between amphotericin B and mannose-PEG-PLGA likely brought about such a significant improvement in the drug encapsulation efficiency.
8.2. Drug-Polymer Complexation. Forming a drug-polymer complex can be beneficial for drug stabilization and improvement in its encapsulation efficiency. Ribeiro et al. used polyL-lysine (PLL) based dendrons to condense pRedN-1 DNA (7.5 kbp), a fluorescent protein vector [67]. When pRedN1 DNA was encapsulated in the form of dendriplexes into PLGA nanoparticles via a $\mathrm{w}_{1} / \mathrm{o} / \mathrm{w}_{2}$ emulsion technique, DNA encapsulation efficiency amounted to $15.6 \%$, higher than that observed with the uncomplexed DNA (9.9\%). A similar tactic was attempted elsewhere: mIL-18 plasmid DNA (pC1-mIL18) was first condensed by a cationic PLL, and the PLLDNA complex was then encapsulated in PEG-PLGA-PEG nanoparticles via a $\mathrm{w}_{1} / \mathrm{o} / \mathrm{w}_{2}$ emulsion technique [10]. The authors demonstrated that the polyplex system maintained DNA integrity during nanoencapsulation. In the work of Vasudev et al., PEGylation was used to stabilize proteins against harsh conditions that they might encounter during an emulsion-based nanoencapsulation process [148]. They demonstrated that the PEGylated asparaginase was more stable than the native protein when exposed to an organic solvent and sonication.

8.3. Control of Continuous Phase. During a $\mathrm{w}_{1} / \mathrm{o} / \mathrm{w}_{2}$ nanoencapsulation process, addition of electrolytes or nonelectrolytes to a $\mathrm{w}_{2}$ phase helps decrease a difference in osmotic pressure between $\mathrm{w}_{1}$ and $\mathrm{w}_{2}$ phases, thereby retarding the leaching of a hydrophilic drug to the $\mathrm{w}_{2}$ phase. This helps improve drug encapsulation efficiency. Zhang et al. encapsulated melanoma antigen peptides (TRP2, hgp100, or p15E) into PLGA nanoparticles via a $\mathrm{w}_{1} / \mathrm{o} / \mathrm{w}_{2}$ emulsion solvent evaporation method [62]. The encapsulation efficiencies of TRP2 and hgp100 were $10.2 \pm 2.1$ and $0.5 \pm 0.2 \%$, respectively. When $5 \%$ of $\mathrm{NaCl}$ was added to the $\mathrm{w}_{2}$ phase, their corresponding values were increased to $39.6 \pm 5.6$ and $7.7 \pm 2.2 \%$. Using a $\mathrm{w}_{2}$ phase containing $15 \%$ glucose also contributed to obtaining better encapsulation efficiency for each peptide. Even though the authors reported that $5 \% \mathrm{NaCl}$ provided better encapsulation efficiency than $15 \%$ glucose, they did not elaborate on this difference. As far as the osmotic pressure is concerned, $1 \mathrm{~g}$ of glucose is equivalent to $0.18 \mathrm{~g}$ of $\mathrm{NaCl}$. As a consequence, $15 \%$ glucose might not provide the same osmotic pressure effect as $5 \% \mathrm{NaCl}$ does.

Cohen et al. encapsulated plasmid DNA (7.3 kbp) into PLGA nanoparticles via the $\mathrm{w}_{1} / \mathrm{o} / \mathrm{w}_{2}$ emulsion technique, and the measured plasmid DNA encapsulation efficiency was $27.9 \pm 1.0 \%$ [55]. Adding $\mathrm{CaCl}_{2}$ into the $\mathrm{w}_{2}$ phase resulted in the enhancement of the plasmid DNA encapsulation efficiency to $73.8 \pm 21.3 \%$. A similar strategy, that is, controlling the osmotic pressure gradient between $\mathrm{w}_{1}$ and $\mathrm{w}_{2}$ phases, was also suggested to be useful in minimizing the loss of hydrophilic immunogens during nanoencapsulation [149].

Drug solubility in a continuous phase is also closely related to drug encapsulation efficiency. The solubility of drugs with ionizable groups is greatly influenced by the solution $\mathrm{pH}$. For example, the aqueous solubility of basic compounds (e.g., Rhodamine 123) decreases with increasing $\mathrm{pH}$. Therefore, alkalinizing the aqueous phase minimizes their aqueous solubility and helps inhibit drug diffusion 
from emulsion droplets/nascent nanoparticles to an aqueous continuous phase [150].

8.4. Optimization of $w_{1}$ Composition for $w_{1} / o / w_{2}$ Emulsion. Cun et al. encapsulated green fluorescent protein (GFP) siRNA into PLGA nanoparticles via a $\mathrm{w}_{1} / \mathrm{o} / \mathrm{w}_{2}$ emulsion technique [61]. Dissolving acetylated BSA in the $\mathrm{w}_{1}$ phase affected the siRNA encapsulation efficiency to a great extent. At an acetylated BSA : siRNA ratio of $4: 1$, there was almost 2 -fold increase in the siRNA encapsulation efficiency, in comparison to the case of using an acetylated BSA-free $\mathrm{w}_{1}$ phase $(46.0 \pm 8.7 \%$ versus $26.1 \pm 0.1 \%)$. It is known that a stable emulsion helps reduce the leakage of hydrophilic drugs from $\mathrm{w}_{1}$ to $\mathrm{w}_{2}$ phases during solvent removal [151]. Protein molecules are surface active that they are involved in stabilization of emulsions. This principle may be held accountable for the improved encapsulation efficiency of siRNA in the presence of acetylated BSA. Varshochian also demonstrated the stabilizing effect of albumins upon the activity of a monoclonal antibody, bevacizumab. The copresence with $10 \%$ BSA or HSA in the $\mathrm{w}_{1}$ phase preserved its activity up to $90 \%$ during emulsification [48]. In the practice of a $\mathrm{w}_{1} / \mathrm{o} / \mathrm{w}_{2}$ emulsion procedure, an increase in viscosity of the $\mathrm{w}_{1}$ phase also helps improve drug encapsulation efficiency. For example, Cheng et al. added carboxymethyl cellulose into the $\mathrm{w}_{1}$ phase, in order to improve the encapsulation efficiency of cisplatin into PLGA-PEG nanoparticles [152].

8.5. Development of New Nanoencapsulation Technology Platforms. As discussed before, HIP-hybridized o/w solvent evaporation/extraction method can significantly improve the encapsulation efficiency of hydrophilic drugs, compared to a typical $\mathrm{w}_{1} / \mathrm{o} / \mathrm{w}_{2}$ emulsion method. Other types of technology platforms are being developed to rapidly generate PLGA nanoparticles and to improve drug encapsulation efficiency. A microfluidic technique such as flow focusing is an exemplary case. Martín-Banderas et al. used this flow focusing technique to encapsulate gemcitabine, a hydrophilic drug, into PLGA particles [138]. As control, they prepared PLGA particles via a typical $\mathrm{w}_{1} / \mathrm{o} / \mathrm{w}_{2}$ emulsion technique. The encapsulation efficiency of gemcitabine attained with the $\mathrm{w}_{1} / \mathrm{o} / \mathrm{w}_{2}$ emulsion technique was $57.5 \%$, but that observed with the flow focusing was $97.3 \%$. In general, the flow focusing technique allows the rapid preparation of PLGA nanoparticles in a single-step, which contributes to the minimization of drug loss.

\section{Special Mixing Devices}

9.1. Static Mixers. Typical mixers used for nanoencapsulation include high-shear homogenizers (e.g., rotor/stator homogenizer), high-energy sonifiers (e.g., probe sonicator), and highpressure homogenizers (e.g., microfluidizer). These mixing devices entail unwanted effects for the structural integrity of fragile biomacromolecules, thereby triggering their denaturation, aggregation, and/or degradation. They also pose additional limitations. While high-shear homogenizers allow the preparation of emulsions with droplet sizes of $2 \sim 5 \mu \mathrm{m}$, it is quite difficult to prepare submicron-sized emulsions made of two immiscible solvents (e.g., methylene chloride and water). Sonication can present the risks of shedding titanium particles out of its probe tip and oxidizing lipidic materials constituting lipid-PLGA hybrid nanoparticles. Also, highpressure homogenizers yield relatively low energy efficiency, because much of energy input is lost as heat.

Static mixers have been sought to overcome the obstacles encountered with traditional mixing devices. Static mixers have a series of mixing elements that are arrayed inside a tube, a pipe, or a column $[153,154]$. Since static mixers have no moving parts, they are known as motionless mixers. External pumps are used to move liquid streams through helically- or blade-designed mixing elements. Inside static mixers, liquid streams undergo flow division, radial momentum transfer, and inertia reversal. As a consequence, static mixers can create very fine dispersions or stable emulsions with uniform sizes [155]. A number of static mixers are commercially available in the market: Ross motionless mixers, Admixer inline static mixers, Koflo static mixers, Chemineer Kenics KM static mixers, JLS Samhwa static mixers, and Sulzer SMX mixers.

Static mixers are utilized for the preparation of PLGA microspheres through emulsion-based microencapsulation processes [156-160]. They are also used to generate primary and/or secondary emulsions, to reduce the size of pre-mix emulsion droplets, or to accelerate the process of solvent removal [161-163]. Their application for preparing PLGA nanoparticles via nanoprecipitation is disclosed in United States patent US 8618240 [163]. A Koflo static mixer (5 mm outer diameter, 12 24 polyacetal helical mixing elements) was extended through a Y-shaped tubing connector: one arm was used for the flow of an aqueous PVA solution, and the other arm was utilized for flowing 1.25\% PLGA in acetone solution. Two gear pumps were used to drive the polymeric dispersed phase and the antisolvent into the static mixing device. Solvent mixing and diffusion left an instant generation of nanoparticles. When the combined flow rate of the two liquids varied from 26 to $438 \mathrm{~mL} / \mathrm{min}$ (the flow rate ratio of the antisolvent to the polymeric dispersed phase $=$ $5: 1)$, the average nanoparticle size ranged from 180 to $84 \mathrm{~nm}$ (Figure 4).

Attributes of a static mixer (e.g., its diameter and length, design, and number of mixing elements) can be manipulated to prepare nanoparticles with a targeted size distribution. Static mixers tend to produce emulsion droplets with narrower size distributions than do mechanical agitators. Other merits of static mixers over typical mechanical mixers include uniform shear across whole liquid streams, short residence time of liquid streams inside the mixers, predictable performance, and easy adaptation for scale-up. In particular, static mixers use significantly less energy to generate emulsion droplets than high-shear/high-energy/high-pressure homogenizers [164]. Furthermore, static mixers can be easily dissembled for cleaning, maintenance, and sterilization.

9.2. Other Mixing Devices. A Total Recirculation OneMachine System (TROMS) mixing device was proposed to generate a $\mathrm{w}_{1} / \mathrm{o} / \mathrm{w}_{2}$ emulsion [165]. This mixing device was reported to ensure the safe loading of plasmid DNA into 


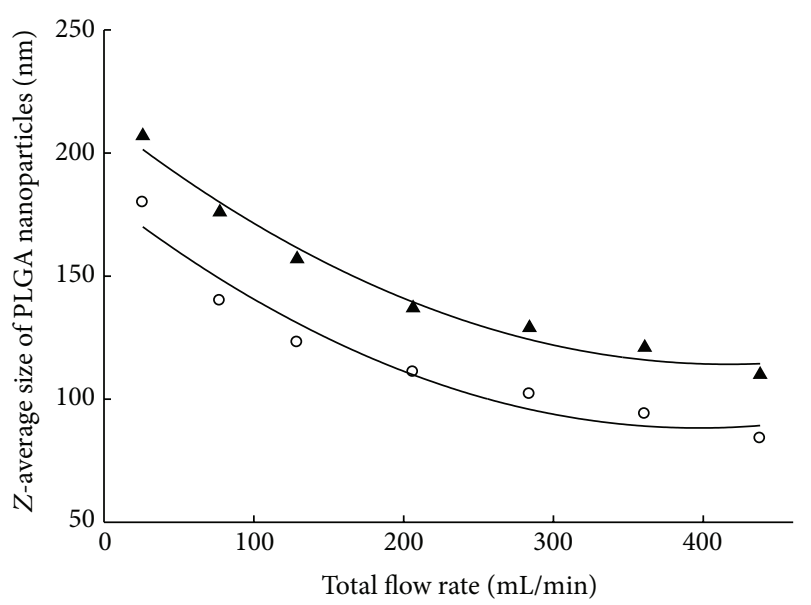

Figure 4: Dependence of PLGA nanoparticle size upon the combined flow rate of a PLGA dispersed phase and an aqueous solution. A PLGA concentration in the dispersed phase was either (०) 1.25 or (ム) $2 \%$.

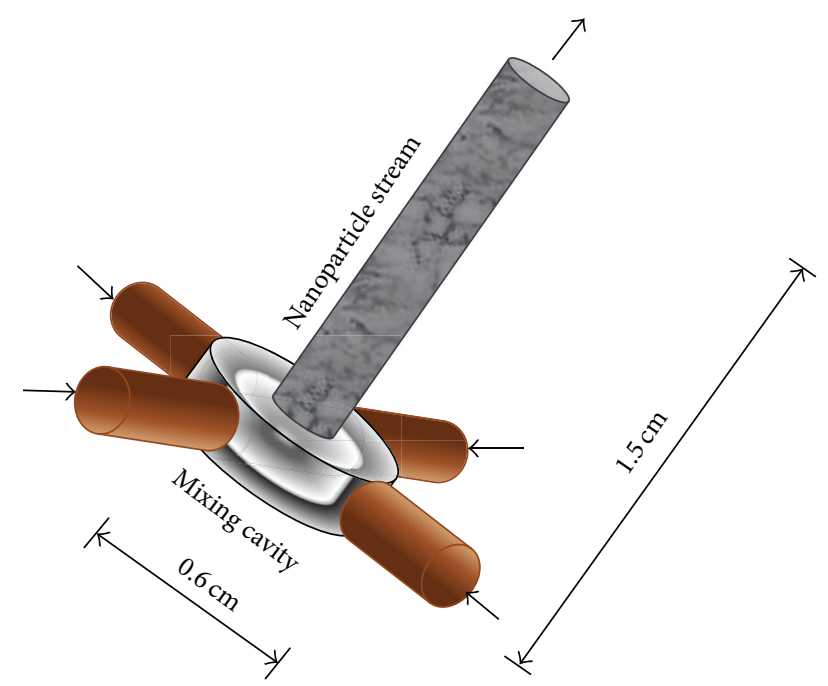

FIGURE 5: Dimension of a typical MIVM composed of inlets, mixing chamber, and outlet.

PLGA microspheres. Santos et al. also used the TROMS to produce PLGA nanoparticles loaded with a kinetoplastid membrane protein that could induce an effective immune response against leishmaniasis [166].

A multi-inlet vortex mixer (MIVM) was suggested elsewhere to prepare polymeric nanoparticles following the principle of nanoprecipitation [167-169]. As shown in Figure 5, the MIVM consists of 4 inlets, one main mixing cavity, and one outlet. Different liquid streams can be introduced into the MIVM at various input ratios. The mixing device also provides rapid, homogeneous micromixing at high Reynolds number. As the characteristic mixing time of the MIVM is in the scale of milliseconds, nanoparticles are produced quickly. These attributes help not only to narrow down the size distribution of nanoparticles but also to improve drug encapsulation efficiency. For example, in the work of
Cheng et al., two of the inlet streams were water, one was PEG-PLGA and curcumin in dimethyl formamide, and the last was an aqueous polyvinyl pyrrolidone solution [170]. They produced 51 76 nm sized PLGA particles with the drug encapsulation efficiency of nearly $100 \%$. Recently, Shen et al. have also reported the use of MIVM to produce curcuminloaded PLGA nanoparticles [81]. Four streams were used in their mixer: stream 1 was a dispersed phase made of tetrahydrofuran, PLGA, and curcumin; and the other three inlet streams were water. Their nanoparticle size ranged from 79 to $120 \mathrm{~nm}$, and the drug encapsulation efficiencies were between 92.4 and $95.4 \%$.

\section{Preparation of PLGA Nanoparticles with Multiple Drug Loads}

When combination products containing two or more drugs act on the site of interest, they can maximize their effects while minimizing side effects. Delivery systems releasing two drugs at different rates can also enhance their therapeutic efficacy [171]. Based on these underlying principles, many different types of PLGA nanoparticles loaded with multiple drugs have been developed (Figure 6). One common type is PLGA nanoparticles containing multiple water-insoluble drugs in their hydrophobic core (Figure 6(a)). Theranostic PLGA nanoparticles contain both a hydrophobic drug and superparamagnetic iron oxide (SPIO) (Figure 6(b)). Schleich et al. has prepared nanoparticles containing paclitaxel and SPIO following an emulsion solvent evaporation method [172]. These nanoparticles have the potential to become nanocarriers for drug delivery, molecular imaging, and realtime monitoring of therapeutic outcome.

Developing a codelivery system for drugs that have fundamentally dissimilar water-solubility and different mechanisms of action has been an intriguing issue. Doxorubicin and paclitaxel are among the most common anticancer drugs in clinical use. However, they have disparate water solubility (hydrophilic versus hydrophobic) and different modes of action. In such cases, a $\mathrm{w}_{1} / \mathrm{o} / \mathrm{w}_{2}$ emulsion technique can be modified to load both hydrophilic and hydrophobic drugs in PLGA nanoparticles (Figure 6(c)) [173-176]. For example, following a $\mathrm{w}_{1} / \mathrm{o} / \mathrm{w}_{2}$ emulsion nanoencapsulation procedure, Heo et al. nanoencapsulated hydrophilic stat 3 siRNA and hydrophobic immune modifier (imiquimod, R837) into PLGA nanoparticles [173]. The loading amount of stat3 siRNA was $4.8 \mu \mathrm{g} / \mathrm{mg}$ of nanoparticles, and that of R837 was $1.9 \mu \mathrm{g} / \mathrm{mg}$ of nanoparticles. Interestingly, Cui et al. used a solid-in-oil-in-water ( $\mathrm{s} / \mathrm{o} / \mathrm{w})$ emulsion method to encapsulate doxorubicin and paclitaxel into transferrin-conjugated magnetic silica PLGA nanoparticles for the treatment of brain glioma [177].

Even though a relevant reference is not cited here, another possible strategy is to load a hydrophobic drug in the PLGA core and to conjugate a hydrophilic drug onto the PEG domain of PLGA nanoparticles (Figure 6(d)). The morphology of PLGA nanoparticles shown in Figure 6(e) is associated with polyelectrolyte-mediated assembly. Cationic polymers such as chitosan and PEI adhere to the surface of negatively charged PLGA nanoparticles by electrostatic attraction 


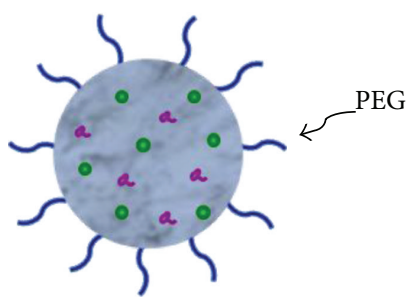

(a)

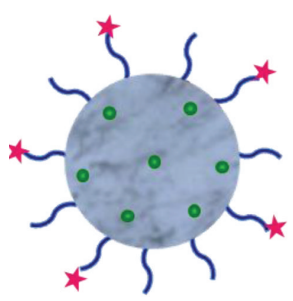

(d)

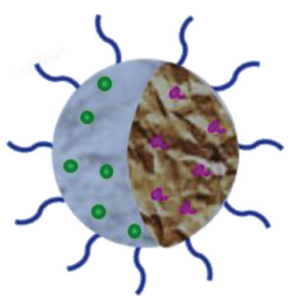

(g)

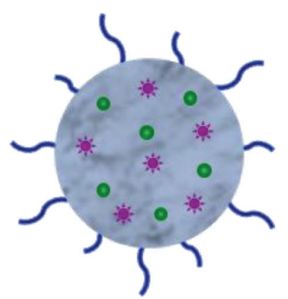

(b)

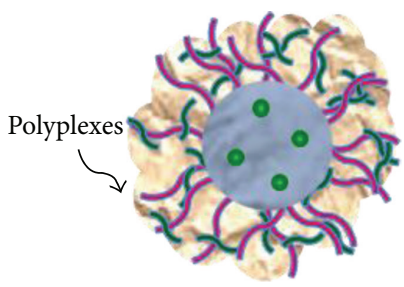

(e)

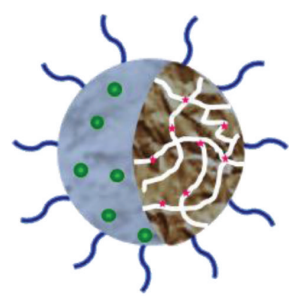

(h)

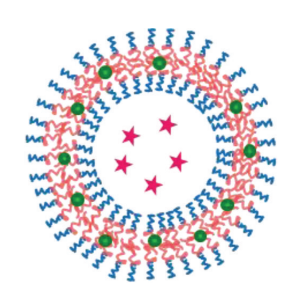

(j)

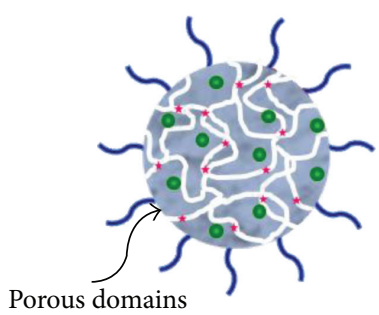

(c)

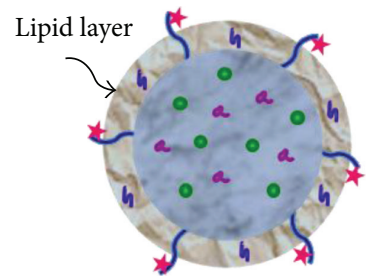

(f)

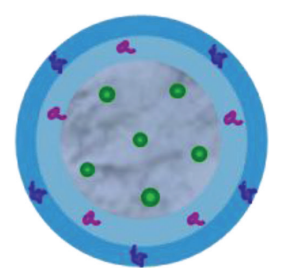

(i)
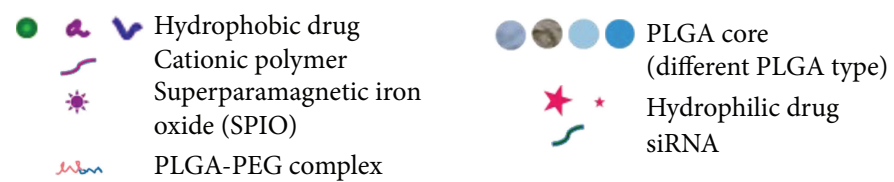

FiguRE 6: Illustrations of various kinds of PLGA-based nanoparticulate systems containing two or more types of drugs: (a) typical nanoparticles; (b) theranostic nanoparticles; (c) porous nanoparticles with both hydrophobic and hydrophilic drugs; (d) drug-spacer-PLGA nanoparticle with a hydrophobic drug in its core; (e) polyplex nanoparticle; (f) core-shell type lipid-PLGA hybrid nanoparticles; (g, h) Janus nanoparticles; (i) onion-type nanoparticles with multiple layers; and (j) polymersomes.

to form an adsorption monolayer. Further adsorption of cationic polymers is driven primarily by hydrogen bonding or van der Waal's force. Such PLGA-cationic polymer nanoparticles can dissolve a hydrophobic drug in their core and are able to complex with negatively charged biomacromolecules on their surface $[92,178-181]$. For example, Su et al. were able to prepare PLGA nanoparticles loaded with paclitaxel, coated by PEI and complexed with stat3 siRNA [92]. Drug-free PLGA nanoparticles had a mean diameter of $87 \mathrm{~nm}$ and zeta potential of $-35.7 \mathrm{mV}$, but those loaded with paclitaxel and the siRNA had the mean diameter of $249 \mathrm{~nm}$ and displayed a net positive charge of $40.8 \mathrm{mV}$. Their in vitro study demonstrated that stat 3 of A549/T12 cancer cells was silenced and the antitumor efficacy of paclitaxel was potentiated. As a concern for the toxicity of PEI exists, the polymer is often substituted with a more biocompatible polymer such as chitosan. Yuan et al. demonstrated that a high encapsulation efficiency of siRNA up to $77.7 \%$ could be achieved, depending on the amount 
of chitosan adsorbed on the surface of PLGA nanoparticles [182]. Other possible cationic polymer candidates are poly-Llysine and poly-allylamine hydrochloride $[183,184]$.

Lipid-PLGA hybrid nanoparticles consisting of a hydrophobic polymeric core and a lipidic shell have been recently suggested as a promising nanoparticulate drug delivery platform [185-187]. Such core-shell type nanoparticles can achieve multiple drug combination therapy and temporal release of more than two drugs (Figure 6(f)). Major components of lipid-PLGA hybrid nanoparticles are PLGA, lipid (e.g., lecithin), and lipid-PEG (e.g., DSPE-PEG). Hydrophobic drugs are loaded into the PLGA hydrophobic core and/or lipid layer, while water-soluble drugs are either adsorbed onto the hydrophilic corona or conjugated to the component of the hydrophilic corona. For example, doxorubicin and a photothermal agent (indocyanine green, ICG) were loaded in lipid-PLGA hybrid nanoparticles, in order to deliver the two agents simultaneously to a tumor region [188]. The nanoparticles not only synergistically induced the death of both doxorubicin-sensitive and doxorubicin-resistant cancer cells in vitro, but also suppressed the tumor growth and recurrence in vivo. Lipid-PLGA hybrid nanoparticles containing both doxorubicin and combretastatin were also introduced, in order to target tumor cells and neovasculature at the same time [189]. A doxorubicin-PLGA conjugate was used to fabricate nanoparticles, which was then added to liposomes consisting of DSPE-PEG, phosphatidylcholine, cholesterol, and combretastatin A4. Extruding the mixture led to the production of a "nanocell" architecture consisting of a nuclear nanoparticle and an extranuclear lecithin/PEGylated lipid envelope. When used in a murine tumor model, combretastatin A4 was first released to inhibit the growth of tumor blood vessels. The breakdown of the doxorubicin-PLGA conjugate provided sustained release of doxorubicin to kill tumor cells. Finally, Huang et al. prepared aptamer sgc8-coated core-shell type PLGA hybrid nanoparticles coencapsulating paclitaxel and doxorubicin [190].

Janus PLGA nanoparticles with bicompartmental morphology are shown in Figures 6(g) and 6(h) [93, 132]. Janus nanoparticles allow coencapsulation of dual drugs with fundamentally different water solubility, as described earlier (Figure 6(h)). They also permit the controlled release of multiple drugs in a specific order with tailored release rates. Onion-like PLGA nanoparticles with multiple layers can be prepared by electrohydrodynamic atomisation (EHDA) techniques (Figure 6(i)). EHDA refers to a process that uses electrical forces to break a liquid jet into tiny droplets [191]. Labbaf et al. designed a coaxial EHDA system using three needles, in order to prepare triple-layered polymeric particles consisting of PLGA, polycaprolactone, and polymethylsilsesquioxane [192]. Such a coaxial EHDA processing enables encapsulation of different drugs inside each polymeric matrix in a single step, leading to the production of onion-like nanoparticles.

Finally, polymersomes can be utilized for coencapsulating multiple drugs (Figure 6(j)). Polymersomes are bilayer vesicles made from self-assembling amphiphilic copolymers such as PEG-PLGA. The self-assembling dynamics are determined by the characteristics of their constituents, as described elsewhere [4]. In comparison to liposomes, polymersomes have more a PEGylated ingredient, more densely packed PEG brush on their surface, and a thicker bilayer membrane [193]. Polymersomes can enable drug cocktail therapy that delivers multiple drugs to single or multiple cellular targets. Based on this tactic, Ahmed et al. used PEG- polybutadiene and PEGPLGA as building blocks for polymersomes [194]. Sonication, freezing-thawing, and extrusion cycles were conducted to coencapsulate doxorubicin and paclitaxel into the vesicle-like nanoparticles in the size of $100 \mathrm{~nm}$. Cryo-TEM revealed that the thickness of the vesicle bilayer was 10 14 nm.

In addition to the PLGA-based nanoparticles shown in Figure 6, innovative approaches are being made to load either multiple drugs or a drug along with a diagnostic agent into various kinds of nanoparticles. Relevant examples include smart drug delivery core-shell nanoparticles, multiple shells, chamber particles, and the like. An excellent review on this topic has been recently published elsewhere [195].

\section{Consideration of Solvent Type}

Methylene chloride and chloroform are frequently used to prepare PLGA microparticles and nanoparticles. However, they are hazardous substances with carcinogenic and tumorigenic properties. The United States EPA and OSHA categorize them as probable human carcinogens. Also, they are ozone-depleting halogenated solvents that increase the risk of human skin cancers. The International Conference on Harmonisation ( $\mathrm{ICH}$ ) categorizes them as Class 2 solvents [196]. Due to these properties, their residual contents in drug products are tightly regulated by the FDA. For example, the limit levels of residual chloroform and methylene chloride in finished drug products are 60 and 600 ppm, respectively. Acetonitrile, $N$-methylpyrrolidone, tetrahydrofuran, and toluene are used for nanoprecipitation as well, but they are also classified as Class 2 solvents. Class 3 solvents with low toxic potential are favored in the pharmaceutical industry. Representative examples of Class 3 solvents used for the preparation of PLGA particles are acetone, ethyl acetate, butyl acetate, isopropyl formate, and glycofurol [197-206]. As shown in Table 3, acetone is the most predominantly used solvent for nanoprecipitation, but it exerts poor solvation power for high-molecular-weight PLGA polymers and certain hydrophobic drugs. Ethyl acetate has been accepted as an alternative to methylene chloride and chloroform in the practice of emulsion solvent evaporation/extraction processes. However, its slight water miscibility and low volatility present some challenging issues with regard to emulsification, solvent removal, and product yield [198, 207, 208].

Surprisingly, there are few reports dealing with residual solvents in PLGA nanoparticles. Residual solvents play important role on their lyophilization, reconstitution in an aqueous vehicle, and syringeability. Therefore, their residual contents in PLGA nanoparticles should be analyzed by validated analytical methodologies such as thermogravimetric analysis and head-space gas chromatography equipped with a flame ionization detector or a mass spectrometer [209]. According to regulatory specifications, the residual level of an ICH Class 3 solvent in drug products should 
be less than $5000 \mathrm{ppm}$. In addition, a solvent used for nanoencapsulation should be evaluated in terms of solubility parameter, polymer-solvent interaction parameter, and drugsolvent thermodynamic property. Therefore, research aiming at finding alternative solvents for nanoencapsulation deserves more attention.

\section{Challenges to Product Development of PLGA Nanoparticles}

On a laboratory scale, there are various options available for the preparation of PLGA nanoparticles by emulsion-based technology platforms (Table 4). Different nanoencapsulation methods lead to the formation of PLGA nanoparticles with different qualities. Key aspects discussed from the manufacturing perspective are size uniformity of nanoparticle populations, drug encapsulation efficiency, mixing device, solvent type, solvent residue in nanoparticles, and mixing device. Other foremost considerations that should be given to product development include scalability of a nanoencapsulation process, reproducibility, nanoparticle characterization, and quality control. Also considered as formidable hurdles are modulating nanoparticle functionality, programmability, and targetability; adjusting drug release rate with minimal burst; and correlating in vitro-in vivo behavior of nanoparticles. Relevant issues have been well discussed elsewhere $[11,210,211]$.

\section{Summary and Conclusions}

Even though PLGA nanoparticles have already demonstrated great potentials as drug carriers, many overwhelming tasks still remain to be solved for their clinical translation. Before rushing into the investigation of in vivo behaviors of PLGA nanoparticles, it is imperative to make an in-depth assessment of technological aspects of a nanoencapsulation method of choice. It is also critical to carefully assess the quality of nanoparticles. In the practice of general emulsion-templated nanoencapsulation processes, mixing devices create zones of energy density in which high pressure, shear, turbulence, and/or cavitation drive the breakups of large emulsion droplets into nanosized ones. High-energy, high-shear, and high-pressure mixing devices damage the structural integrity of fragile macromolecules. Preparing PLGA nanoparticles with high loads of hydrophilic drugs often remains challenging. In addition, the mixing of a polymeric dispersed phase with antisolvent follows a bulk process, which lacks the ability to precisely direct the process of emulsification and the rate of PLGA precipitation. As a result, this procedure gives rise to the formation of a heterogeneous nanoparticle population with regard to mean diameter, size distribution, morphology, and drug content. Therefore, selecting or developing a nanoencapsulation method must surmount the aforementioned obstacles. At the same time, the nanoencapsulation method should be able to produce nanoparticles that meet the desired standards in a consistent manner.
Nowadays, new nanoencapsulation technologies based on microfluidic mixing devices, static mixers, porous membrane mixers, and flow focusing nozzles are being developed in attempt to mitigate the aforementioned concerns. These technologies can be further adjusted to prepare novel nanoparticle composites such as Janus nanoparticles that can accommodate multiple drugs and manipulate their release kinetics. Relevant technologies will evolve in the coming days, so as to continuously expand diverse applications of PLGA-based particulate carriers in the field of nanomedicine.

\section{Conflict of Interests}

The authors report no conflict of interests regarding the publication of this paper.

\section{Acknowledgment}

This study was supported by the Korea SGER Program through the National Research Foundation of Korea (NRF) funded by the Ministry of Education, Science and Technology (NRF-2014R1A1A2A16054899).

\section{References}

[1] R. Bawa, "Nanoparticle-based therapeutics in humans: a survey," Nanotechnology Law and Business, vol. 5, no. 2, pp. 135-155, 2008.

[2] J. M. Chan, P. M. Valencia, L. Zhang, R. Langer, and O. C. Farokhzad, "Polymeric nanoparticles for drug delivery," in Cancer Nanotechnology, vol. 624 of Methods in Molecular Biology, pp. 163-175, Humana Press, 2010.

[3] H. K. Makadia and S. J. Siegel, "Poly lactic-co-glycolic acid (PLGA) as biodegradable controlled drug delivery carrier," Polymers, vol. 3, no. 3, pp. 1377-1397, 2011.

[4] H. Sah, L. A. Thoma, H. R. Desu, E. Sah, and G. C. Wood, "Concepts and practices used to develop functional PLGAbased nanoparticulate systems," International Journal of Nanomedicine, vol. 8, pp. 747-765, 2013.

[5] V. Sanna, N. Pala, and M. Sechi, "Targeted therapy using nanotechnology: focus on cancer," International Journal of Nanomedicine, vol. 9, no. 1, pp. 467-483, 2014.

[6] C. Pinto Reis, R. J. Neufeld, A. J. Ribeiro, and F. Veiga, "Nanoencapsulation I. Methods for preparation of drug-loaded polymeric nanoparticles," Nanomedicine: Nanotechnology, Biology, and Medicine, vol. 2, no. 1, pp. 8-21, 2006.

[7] F. Danhier, E. Ansorena, J. M. Silva, R. Coco, A. Le Breton, and V. Préat, "PLGA-based nanoparticles: an overview of biomedical applications," Journal of Controlled Release, vol. 161, no. 2, pp. 505-522, 2012.

[8] B. Jeong, Y. H. Bae, and S. W. Kim, "In situ gelation of PEG-PLGA-PEG triblock copolymer aqueous solutions and degradation thereof," Journal of Biomedical Materials Research, vol. 50, no. 2, pp. 171-177, 2000.

[9] J. Cheng, B. A. Teply, I. Sherifi et al., "Formulation of functionalized PLGA-PEG nanoparticles for in vivo targeted drug delivery," Biomaterials, vol. 28, no. 5, pp. 869-876, 2007.

[10] Y. Nie, Z. R. Zhang, B. He, and Z. Gu, "Investigation of PEGPLGA-PEG nanoparticles-based multipolyplexes for IL-18 gene delivery," Journal of Biomaterials Applications, vol. 26, no. 8, pp. 893-916, 2012. 
[11] I. Bala, S. Hariharan, and M. N. V. R. Kumar, "PLGA nanoparticles in drug delivery: the state of the art," Critical Reviews in Therapeutic Drug Carrier Systems, vol. 21, no. 5, pp. 387-422, 2004.

[12] N. Anton, J.-P. Benoit, and P. Saulnier, "Design and production of nanoparticles formulated from nano-emulsion templates-a review," Journal of Controlled Release, vol. 128, no. 3, pp. 185-199, 2008.

[13] R. Kumar, G. Troiano, J. M. Ramstack, P. Herbert, and M. Figa, “Coacervation process," European Patent EP 2131815, 2012.

[14] M. Nahar and N. K. Jain, "Preparation, characterization and evaluation of targeting potential of amphotericin B-loaded engineered PLGA nanoparticles," Pharmaceutical Research, vol. 26, no. 12, pp. 2588-2598, 2009.

[15] I. Özcan, E. Azizoğlu, T. Şenyiğit, M. Özyazici, and Ö. Özer, "Comparison of PLGA and lecithin/chitosan nanoparticles for dermal targeting of betamethasone valerate," Journal of Drug Targeting, vol. 21, no. 6, pp. 542-550, 2013.

[16] P. Yin, Y. Wang, Y. Y. Qiu et al., "Bufalin-loaded mPEG-PLGAPLL-cRGD nanoparticles: preparation, cellular uptake, tissue distribution, and anticancer activity," International Journal of Nanomedicine, vol. 7, pp. 3961-3969, 2012.

[17] C. Matthäus, S. Schubert, M. Schmitt et al., "Resonance Raman spectral imaging of intracellular uptake of $\beta$ carotene loaded poly(D, L-lactide-co-glycolide) nanoparticles," ChemPhysChem, vol. 14, no. 1, pp. 155-161, 2013.

[18] M. M. Ibrahim, A.-E. H. Abd-Elgawad, O. A.-E. Soliman, and M. M. Jablonski, "Nanoparticle-based topical ophthalmic formulations for sustained celecoxib release," Journal of Pharmaceutical Sciences, vol. 102, no. 3, pp. 1036-1053, 2013.

[19] A. Xu, M. Yao, G. Xu et al., "A physical model for the size-dependent cellular uptake of nanoparticles modified with cationic surfactants," International Journal of Nanomedicine, vol. 7, pp. 3547-3554, 2012.

[20] N. M. Khalil, T. C. F. do Nascimento, D. M. Casa et al., "Pharmacokinetics of curcumin-loaded PLGA and PLGA-PEG blend nanoparticles after oral administration in rats," Colloids and Surfaces B: Biointerfaces, vol. 101, pp. 353-360, 2013.

[21] P. Aksungur, M. Demirbilek, E. B. Denkbaş, and N. Ünlü, "Comparative evaluation of cyclosporine A/HP $\beta C D$ incorporated PLGA nanoparticles for development of effective ocular preparations," Journal of Microencapsulation, vol. 29, no. 6, pp. 605-613, 2012.

[22] Z. Li, K. Liu, P. Sun et al., "Poly(D, L-lactide-co-glycolide)/ montmorillonite nanoparticles for improved oral delivery of exemestane," Journal of Microencapsulation, vol. 30, no. 5, pp. 432-440, 2013.

[23] A. Budhian, S. J. Siegel, and K. I. Winey, "Production of haloperidol-loaded PLGA nanoparticles for extended controlled drug release of haloperidol," Journal of Microencapsulation, vol. 22, no. 7, pp. 773-785, 2005.

[24] C. T. S. Turk, U. C. Oz, T. M. Serim, and C. Hascicek, "Formulation and optimization of nonionic surfactants emulsified nimesulide-loaded PLGA-based nanoparticles by design of experiments," AAPS PharmSciTech, vol. 15, no. 1, pp. 161-176, 2014.

[25] V. Bhardwaj, D. D. Ankola, S. C. Gupta, M. Schneider, C.-M. Lehr, and M. N. V. R. Kumar, "PLGA nanoparticles stabilized with cationic surfactant: safety studies and application in oral delivery of paclitaxel to treat chemical-induced breast cancer in rat," Pharmaceutical Research, vol. 26, no. 11, pp. 2495-2503, 2009.
[26] C. Jin, L. Bai, H. Wu, W. Song, G. Guo, and K. Dou, "Cytotoxicity of paclitaxel incorporated in plga nanoparticles on hypoxic human tumor cells," Pharmaceutical Research, vol. 26, no. 7, pp. 1776-1784, 2009.

[27] S. K. Swaminathan, E. Roger, U. Toti, L. Niu, J. R. Ohlfest, and J. Panyam, "CD133-targeted paclitaxel delivery inhibits local tumor recurrence in amousemodel of breast cancer," Journal of Controlled Release, vol. 171, no. 3, pp. 280-287, 2013.

[28] S. Chowdhury, R. Guha, R. Trivedi, U. B. Kompella, A. Konar, and S. Hazra, "Pirfenidone nanoparticles improve corneal wound healing and prevent scarring following alkali burn," PLoS ONE, vol. 8, no. 8, Article ID e70528, 2013.

[29] R. M. Mainardes and R. C. Evangelista, "PLGA nanoparticles containing praziquantel: effect of formulation variables on size distribution," International Journal of Pharmaceutics, vol. 290, no. 1-2, pp. 137-144, 2005.

[30] P. Liu, Y. Sun, Q. Wang, Y. Sun, H. Li, and Y. Duan, "Intracellular trafficking and cellular uptake mechanism of mPEG-PLGAPLL and mPEG-PLGA-PLL-Gal nanoparticles for targeted delivery to hepatomas," Biomaterials, vol. 35, no. 2, pp. 760-770, 2014.

[31] Y. C. Kuo and H. W. Yu, "Transport of saquinavir across human brain-microvascular endothelial cells by poly(lactideco-glycolide) nanoparticles with surface poly- $(\gamma$-glutamic acid)," International Journal of Pharmaceutics, vol. 416, no. 1, pp. 365-375, 2011.

[32] T.-W. Chung, S.-S. Wang, and W.-J. Tsai, "Accelerating thrombolysis with chitosan-coated plasminogen activators encapsulated in poly-(lactide-co-glycolide) (PLGA) nanoparticles," Biomaterials, vol. 29, no. 2, pp. 228-237, 2008.

[33] Y. Wang, P. Liu, Y. Duan et al., "Specific cell targeting with APRPG conjugated PEG-PLGA nanoparticles for treating ovarian cancer," Biomaterials, vol. 35, no. 3, pp. 983-992, 2014.

[34] B. Sinha, B. Mukherjee, and G. Pattnaik, "Poly-lactide-coglycolide nanoparticles containing voriconazole for pulmonary delivery: in vitro and in vivo study," Nanomedicine, vol. 9, no. 1, pp. 94-104, 2013.

[35] H. Mahdavi, H. Mirzadeh, H. Hamishehkar et al., "The effect of process parameters on the size and morphology of poly $(\mathrm{D}, \mathrm{L}-$ lactide-co-glycolide) micro/nanoparticles prepared by an oil in oil emulsion/solvent evaporation technique," Journal of Applied Polymer Science, vol. 116, no. 1, pp. 528-534, 2010.

[36] T. S. J. Kashi, S. Eskandarion, M. Esfandyari-Manesh et al., "Improved drug loading and antibacterial activity of minocycline-loaded PLGA nanoparticles prepared by solid/ oil/water ion pairing method," International Journal of Nanomedicine, vol. 7, pp. 221-234, 2012.

[37] P. M. Hughes, M. Boix, C. Sarrazin, and M. Do, "Oil-in-oil emulsified polymeric implants containing a hypotensive lipid and prostamide," United States patent US 8445027, May 2013.

[38] E. Allémann, R. Gurny, and E. Doelker, "Preparation of aqueous polymeric nanodispersions by a reversible salting-out process: influence of process parameters on particle size," International Journal of Pharmaceutics, vol. 87, no. 1-3, pp. 247-253, 1992.

[39] E. Allemann, J.-C. Leroux, R. Gurny, and E. Doelker, "In vitro extended-release properties of drug-loaded poly(DL-lactic acid) nanoparticles produced by a salting-out procedure," Pharmaceutical Research, vol. 10, no. 12, pp. 1732-1737, 1993.

[40] H. Ibrahim, C. Bindschaedler, E. Doelker, P. Buri, and R. Gurny, "Aqueous nanodispersions prepared by a salting-out process," International Journal of Pharmaceutics, vol. 87, no. 1-3, pp. 239246, 1992. 
[41] S. Galindo-Rodriguez, E. Allémann, H. Fessi, and E. Doelker, "Physicochemical parameters associated with nanoparticle formation in the salting-out, emulsification-diffusion, and nanoprecipitation methods," Pharmaceutical Research, vol. 21, no. 8, pp. 1428-1439, 2004.

[42] B. Wang, M. Gou, X. Zheng et al., "Co-delivery honokiol and doxorubicin in MPEG-PLA nanoparticles," Journal of Nanoscience and Nanotechnology, vol. 10, no. 7, pp. 4166-4172, 2010.

[43] Y. N. Konan, R. Gurny, and E. Allémann, "Preparation and characterization of sterile and freeze-dried sub-200 nm nanoparticles," International Journal of Pharmaceutics, vol. 233, no. 1-2, pp. 239-252, 2002.

[44] M. Vettor, P. Perugini, S. Scalia et al., "Poly(D,L-lactide) nanoencapsulation to reduce photoinactivation of a sunscreen agent," International Journal of Cosmetic Science, vol. 30, no. 3, pp. 219227, 2008 .

[45] M. Vettor, S. Bourgeois, H. Fessi et al., "Skin absorption studies of octyl-methoxycinnamate loaded poly(D,L-lactide) nanoparticles: estimation of the UV filter distribution and release behaviour in skin layers," Journal of Microencapsulation, vol. 27, no. 3, pp. 253-262, 2010.

[46] S. Gupta, A. Agarwal, N. K. Gupta, G. Saraogi, H. Agrawal, and G. P. Agrawal, "Galactose decorated PLGA nanoparticles for hepatic delivery of acyclovir," Drug Development and Industrial Pharmacy, vol. 39, no. 12, pp. 1866-1873, 2013.

[47] P. Liu, H. Yu, Y. Sun, M. Zhu, and Y. Duan, "A mPEG-PLGAb-PLL copolymer carrier for adriamycin and siRNA delivery," Biomaterials, vol. 33, no. 17, pp. 4403-4412, 2012.

[48] R. Varshochian, M. Jeddi-Tehrani, A. R. Mahmoudi et al., "The protective effect of albumin on bevacizumab activity and stability in PLGA nanoparticles intended for retinal and choroidal neovascularization treatments," European Journal of Pharmaceutical Sciences, vol. 50, no. 3-4, pp. 341-352, 2013.

[49] N. Rescignano, L. Tarpani, R. Tiribuzi et al., "Protein encapsulation in biodegradable polymeric nanoparticles: morphology, fluorescence behaviour and stem cell uptake," Macromolecular Bioscience, vol. 13, no. 9, pp. 1204-1212, 2013.

[50] M. Alonso-Sande, A. des Rieux, V. Fievez et al., "Development of PLGA-mannosamine nanoparticles as oral protein carriers," Biomacromolecules, vol. 14, no. 11, pp. 4046-4052, 2013.

[51] S. H. Lee, Z. Zhang, and S.-S. Feng, "Nanoparticles of poly(lactide)-tocopheryl polyethylene glycol succinate (PLATPGS) copolymers for protein drug delivery," Biomaterials, vol. 28, no. 11, pp. 2041-2050, 2007.

[52] N. Samadi, A. Abbadessa, A. di Stefano et al., "The effect of lauryl capping group on protein release and degradation of poly(d,l-lactic-co-glycolic acid) particles," Journal of Controlled Release, vol. 172, no. 2, pp. 436-443, 2013.

[53] S. J. Fairley, S. R. Singh, A. N. Yilma et al., "Chlamydia trachomatis recombinant MOMP encapsulated in PLGA nanoparticles triggers primarily $\mathrm{T}$ helper 1 cellular and antibody immune responses in mice: a desirable candidate nanovaccine," International Journal of Nanomedicine, vol. 8, pp. 2085-2099, 2013.

[54] C. Colonna, R. Dorati, B. Conti, P. Caliceti, and I. Genta, "Sub-unit vaccine against $S$. aureus-mediated infections: setup of nano-sized polymeric adjuvant," International Journal of Pharmaceutics, vol. 452, no. 1-2, pp. 390-401, 2013.

[55] H. Cohen, R. J. Levy, J. Gao et al., "Sustained delivery and expression of DNA encapsulated in polymeric nanoparticles," Gene Therapy, vol. 7, no. 22, pp. 1896-1905, 2000.
[56] F. Li, J. Sun, H. Zhu, X. Wen, C. Lin, and D. Shi, "Preparation and characterization novel polymer-coated magnetic nanoparticles as carriers for doxorubicin," Colloids and Surfaces B: Biointerfaces, vol. 88, no. 1, pp. 58-62, 2011.

[57] M. Wang, Y. Zhang, J. Feng et al., "Preparation, characterization, and in vitro and in vivo investigation of chitosan-coated poly(d,l-lactide-co-glycolide) nanoparticles for intestinal delivery of exendin-4," International Journal of Nanomedicine, vol. 8, pp. 1141-1154, 2013.

[58] Y. Wang, P. Li, and L. Kong, "Chitosan-modified PLGA nanoparticles with versatile surface for improved drug delivery," AAPS PharmSciTech, vol. 14, no. 2, pp. 585-592, 2013.

[59] S. M. Abdelghany, D. J. Quinn, R. J. Ingram et al., "Gentamicinloaded nanoparticles show improved antimicrobial effects towards Pseudomonas aeruginosa infection," International Journal of Nanomedicine, vol. 7, pp. 4053-4063, 2012.

[60] C. Lecaroz, C. Gamazo, M. J. Renedo, and M. J. Blanco-Prieto, "Biodegradable micro- and nanoparticles as long-term delivery vehicles for gentamicin," Journal of Microencapsulation, vol. 23, no. 7, pp. 782-792, 2006.

[61] D. Cun, C. Foged, M. Yang, S. Frøkjær, and H. M. Nielsen, "Preparation and characterization of poly(dl-lactide-coglycolide) nanoparticles for siRNA delivery," International Journal of Pharmaceutics, vol. 390, no. 1, pp. 70-75, 2010.

[62] Z. Zhang, S. Tongchusak, Y. Mizukami et al., "Induction of antitumor cytotoxic $\mathrm{T}$ cell responses through PLGA-nanoparticle mediated antigen delivery," Biomaterials, vol. 32, no. 14, pp. 3666-3678, 2011.

[63] M. Tafaghodi, A. Khamesipour, and M. R. Jaafari, "Immunization against leishmaniasis by PLGA nanospheres encapsulated with autoclaved Leishmania major (ALM) and CpG-ODN," Parasitology Research, vol. 108, no. 5, pp. 1265-1273, 2011.

[64] R. Coco, L. Plapied, V. Pourcelle et al., "Drug delivery to inflamed colon by nanoparticles: comparison of different strategies," International Journal of Pharmaceutics, vol. 440, no. 1, pp. 3-12, 2013.

[65] M. Zaric, O. Lyubomska, O. Touzelet et al., "Skin dendritic cell targeting via microneedle arrays laden with antigenencapsulated poly-D,L-lactide-co-glycolide nanoparticles induces efficient antitumor and antiviral immune responses," ACS Nano, vol. 7, no. 3, pp. 2042-2055, 2013.

[66] S. Sunoqrot, J. W. Bae, S. E. Jin, R. M. Pearson, Y. Liu, and S. Hong, "Kinetically controlled cellular interactions of polymer-polymer and polymer-liposome nanohybrid systems," Bioconjugate Chemistry, vol. 22, no. 3, pp. 466-474, 2011.

[67] S. Ribeiro, N. Hussain, and A. T. Florence, "Release of DNA from dendriplexes encapsulated in PLGA nanoparticles," International Journal of Pharmaceutics, vol. 298, no. 2, pp. 354-360, 2005.

[68] M. Chaturvedi, Y. Molino, B. Sreedhar, M. Khrestchatisky, and L. Kaczmarek, "Tissue inhibitor of matrix metalloproteinases1 loaded poly(lactic-co-glycolic acid) nanoparticles for delivery across the blood-brain barrier," International Journal of Nanomedicine, vol. 9, no. 1, pp. 575-588, 2014.

[69] M. Afshari, K. Derakhshandeh, and L. Hosseinzadeh, "Characterisation, cytotoxicity and apoptosis studies of methotrexateloaded PLGA and PLGA-PEG nanoparticles," Journal of Microencapsulation, vol. 31, no. 3, pp. 239-245, 2014.

[70] F. Ganachaud and J. L. Katz, "Nanoparticles and nanocapsules created using the ouzo effect: spontaneous emulsification as an alternative to ultrasonic and high-shear devices," ChemPhysChem, vol. 6, no. 2, pp. 209-216, 2005. 
[71] H. Fessi, F. Piusieux, J. P. Devissaguet, N. Ammoury, and S. Benita, "Nanocapsule formation by interfacial polymer deposition following solvent displacement," International Journal of Pharmaceutics, vol. 55, no. 1, pp. R1-R4, 1989.

[72] J. Huang, H. Zhang, Y. Yu et al., "Biodegradable self-assembled nanoparticles of poly(d,l-lactide-co-glycolide)/hyaluronic acid block copolymers for target delivery of docetaxel to breast cancer," Biomaterials, vol. 35, no. 1, pp. 550-566, 2014.

[73] R. Dinarvand, N. Sepehri, S. Manoochehri, and F. Atyabi, "Polylactide-co-glycolide nanoparticles for controlled delivery of anticancer agents," International Journal of Nanomedicine, vol. 6, pp. 877-895, 2011.

[74] A. Kumar and K. K. Sawant, "Application of multiple regression analysis in optimization of anastrozole-loaded PLGA nanoparticles," Journal of Microencapsulation, vol. 31, no. 2, pp. 105-114, 2014.

[75] S. Şimşek, H. Eroğlu, B. Kurum, and K. Ulubayram, "Brain targeting of Atorvastatin loaded amphiphilic PLGA- $b$-PEG nanoparticles," Journal of Microencapsulation, vol. 30, no. 1, pp. 10-20, 2013.

[76] C. P. Reis, N. Martinho, C. Rosado, A. S. Fernandes, and A. Roberto, "Design of polymeric nanoparticles and its applications as drug delivery systems for acne treatment," Drug Development and Industrial Pharmacy, vol. 40, no. 3, pp. 409417, 2014.

[77] M. E. Ali and A. Lamprecht, "Polyethylene glycol as an alternative polymer solvent for nanoparticle preparation," International Journal of Pharmaceutics, vol. 456, no. 1, pp. 135-142, 2013.

[78] A. Paul, S. Das, J. Das, A. Samadder, and A. R. KhudaBukhsh, "Cytotoxicity and apoptotic signalling cascade induced by chelidonine-loaded PLGA nanoparticles in HepG2 cells in vitro and bioavailability of nano-chelidonine in mice in vivo," Toxicology Letters, vol. 222, no. 1, pp. 10-22, 2013.

[79] C. S. de Paula, A. C. Tedesco, F. L. Primo, J. M. C. Vilela, M. S. Andrade, and V. C. F. Mosqueira, "Chloroaluminium phthalocyanine polymeric nanoparticles as photosensitisers: photophysical and physicochemical characterisation, release and phototoxicity in vitro," European Journal of Pharmaceutical Sciences, vol. 49, no. 3, pp. 371-381, 2013.

[80] Z. H. Wang, Z. Y. Wang, C. S. Sun, C. Y. Wang, T. Y. Jiang, and S. L. Wang, "Trimethylated chitosan-conjugated PLGA nanoparticles for the delivery of drugs to the brain," Biomaterials, vol. 31, no. 5, pp. 908-915, 2010.

[81] H. Shen, X. Hu, M. Szymusiak, Z. J. Wang, and Y. Liu, "Orally administered nanocurcumin to attenuate morphine tolerance: comparison between negatively charged PLGA and partially and fully PEGylated nanoparticles," Molecular Pharmaceutics, vol. 10, no. 12, pp. 4546-4551, 2013.

[82] W. Tao, X. Zeng, T. Liu et al., "Docetaxel-loaded nanoparticles based on star-shaped mannitol-core PLGA-TPGS diblock copolymer for breast cancer therapy," Acta Biomaterialia, vol. 9, no. 11, pp. 8910-8920, 2013.

[83] T. Musumeci, C. A. Ventura, I. Giannone et al., "PLA/PLGA nanoparticles for sustained release of docetaxel," International Journal of Pharmaceutics, vol. 325, no. 1-2, pp. 172-179, 2006.

[84] C. W. Gan and S. S. Feng, "Transferrin-conjugated nanoparticles of Poly(lactide)-D- $\alpha$-tocopheryl polyethylene glycol succinate diblock copolymer for targeted drug delivery across the blood-brain barrier," Biomaterials, vol. 31, no. 30, pp. 7748-7757, 2010.

[85] L. Martín-Banderas, J. Alvarez-Fuentes, M. Durán-Lobato et al., "Cannabinoid derivate-loaded PLGA nanocarriers for oral administration: formulation, characterization, and cytotoxicity studies," International Journal of Nanomedicine, vol. 7, pp. 57935806, 2012.

[86] H. Y. Kwon, J. Y. Lee, S. W. Choi, Y. Jang, and J. H. Kim, "Preparation of PLGA nanoparticles containing estrogen by emulsification-diffusion method," Colloids and Surfaces A: Physicochemical and Engineering Aspects, vol. 182, no. 1-3, pp. 123-130, 2001.

[87] E. Vega, M. A. Egea, A. C. Calpena, M. Espina, and M. L. García, "Role of hydroxypropyl- $\beta$-cyclodextrin on freezedried and gamma-irradiated PLGA and PLGA-PEG diblock copolymer nanospheres for ophthalmic flurbiprofen delivery," International Journal of Nanomedicine, vol. 7, pp. 1357-1371, 2012.

[88] Y. C. Chen, W. Y. Hsieh, W. F. Lee, and D. T. Zeng, "Effects of surface modification of PLGA-PEG-PLGA nanoparticles on loperamide delivery efficiency across the blood-brain barrier," Journal of Biomaterials Applications, vol. 27, no. 7, pp. 909-922, 2013.

[89] T. Musumeci, C. Bucolo, C. Carbone, R. Pignatello, F. Drago, and G. Puglisi, "Polymeric nanoparticles augment the ocular hypotensive effect of melatonin in rabbits," International Journal of Pharmaceutics, vol. 440, no. 2, pp. 135-140, 2013.

[90] S. S. Bhattacharyya, S. Paul, A. De et al., "Poly(lactide-coglycolide) acid nanoencapsulation of a synthetic coumarin: cytotoxicity and bio-distribution in mice, in cancer cell line and interaction with calf thymus DNA as target," Toxicology and Applied Pharmacology, vol. 253, no. 3, pp. 270-281, 2011.

[91] H.-J. Jeon, Y.-I. Jeong, M.-K. Jang, Y.-H. Park, and J.-W. $\mathrm{Nah}$, "Effect of solvent on the preparation of surfactant-free poly(DL-lactide-co-glycolide) nanoparticles and norfloxacin release characteristics," International Journal of Pharmaceutics, vol. 207, no. 1-2, pp. 99-108, 2000.

[92] W. P. Su, F. Y. Cheng, D. B. Shieh, C. S. Yeh, and W. C. Su, "PLGA nanoparticles codeliver paclitaxel and Stat 3 siRNA to overcome cellular resistance in lung cancer cells," International Journal of Nanomedicine, vol. 7, pp. 4269-4283, 2012.

[93] H. Xie, Z.-G. She, S. Wang, G. Sharma, and J. W. Smith, "One-step fabrication of polymeric Janus nanoparticles for drug delivery," Langmuir, vol. 28, no. 9, pp. 4459-4463, 2012.

[94] V. Bojat, V. Balabanyan, and R. Alyautdin, "The entrapment of paclitaxel in PLGA nanoparticles increases its cytotoxicity against multiresistant cell line," British Journal of Medicine and Medical Research, vol. 1, no. 4, pp. 306-319, 2011.

[95] C. M. Moraes, A. P. de Matos, R. de Lima, A. H. Rosa, E. de Paula, and L. F. Fraceto, "Initial development and characterization of PLGA nanospheres containing ropivacaine," Journal of Biological Physics, vol. 33, no. 5-6, pp. 455-461, 2007.

[96] Y.-I. Jeong, Y.-H. Shim, C. Kim, G.-T. Lim, K.-C. Choi, and C. Yoon, "Effect of cryoprotectants on the reconstitution of surfactant-free nanoparticles of poly(DL-lactide-co-glycolide)," Journal of Microencapsulation, vol. 22, no. 6, pp. 593-601, 2005.

[97] J. Ravindran, H. B. Nair, B. Sung, S. Prasad, R. R. Tekmal, and B. B. Aggarwal, "Thymoquinone poly(lactide-coglycolide) nanoparticles exhibit enhanced anti-proliferative, anti-inflammatory, and chemosensitization potential," Biochemical Pharmacology, vol. 79, no. 11, pp. 1640-1647, 2010.

[98] J. Lalani, M. Rathi, M. Lalan, and A. Misra, "Protein functionalized tramadol-loaded PLGA nanoparticles: preparation, optimization, stability and pharmacodynamic studies," Drug Development and Industrial Pharmacy, vol. 39, no. 6, pp. 854864, 2013. 
[99] A. M. Paiva, R. A. Pinto, M. Teixeira et al., "Development of noncytotoxic PLGA nanoparticles to improve the effect of a new inhibitor of p53-MDM2 interaction," International Journal of Pharmaceutics, vol. 454, no. 1, pp. 394-402, 2013.

[100] S. A. Vitale and J. L. Katz, "Liquid droplet dispersions formed by homogeneous liquid-liquid nucleation: 'the ouzo effect," Langmuir, vol. 19, no. 10, pp. 4105-4110, 2003.

[101] T. Niwa, H. Takeuchi, T. Hino, N. Kunou, and Y. Kawashima, "Preparations of biodegradable nanospheres of water-soluble and insoluble drugs with D,L-lactide/glycolide copolymer by a novel spontaneous emulsification solvent diffusion method, and the drug release behavior," Journal of Controlled Release, vol. 25, no. 1-2, pp. 89-98, 1993.

[102] M. H. El-Shabouri, "Positively charged nanoparticles for improving the oral bioavailability of cyclosporin-A," International Journal of Pharmaceutics, vol. 249, no. 1-2, pp. 101-108, 2002.

[103] H. Murakami, M. Kobayashi, H. Takeuchi, and Y. Kawashima, "Preparation of poly(DL-lactide-co-glycolide) nanoparticles by modified spontaneous emulsification solvent diffusion method," International Journal of Pharmaceutics, vol. 187, no. 2 , pp. 143-152, 1999.

[104] T. Nakashima, M. Shimizu, and M. Kukizaki, "Membrane emulsification by microporous glass," Key Engineering Materials, vol. 61-62, pp. 513-516, 1992.

[105] T. Nakashima, M. Shimizu, and M. Kukizaki, "Particle control of emulsion by membrane emulsification and its applications," Advanced Drug Delivery Reviews, vol. 45, no. 1, pp. 47-56, 2000.

[106] T. Hwang, T. J. Park, W. G. Koh, I. W. Cheong, S. W. Choi, and J. H. Kim, "Fabrication of nano-scale liposomes containing doxorubicin using Shirasu porous glass membrane," Colloids and Surfaces A: Physicochemical and Engineering Aspects, vol. 392, no. 1, pp. 250-255, 2011.

[107] G. H. Ma, M. Nagai, and S. Omi, "Preparation of uniform poly(lactide) microspheres by employing the Shirasu Porous Glass (SPG) emulsification technique," Colloids and Surfaces A: Physicochemical and Engineering Aspects, vol. 153, no. 1-3, pp. 383-394, 1999.

[108] H. Oha, P. Balakrishnana, Y. K. Oh, D. D. Kim, C. S. Yon, and H. G. Choi, "Effect of process parameters on nanoemulsion droplet size and distribution in SPG membrane emulsification," International Journal of Pharmaceutics, vol. 404, pp. 191-197, 2011.

[109] W. Liu, X.-L. Yang, and W. S. W. Ho, "Preparation of uniformsized multiple emulsions and micro/nano particulates for drug delivery by membrane emulsification," Journal of Pharmaceutical Sciences, vol. 100, no. 1, pp. 75-93, 2011.

[110] C. Charcosset, I. Limayem, and H. Fessi, "The membrane emulsification process-a review," Journal of Chemical Technology and Biotechnology, vol. 79, no. 3, pp. 209-218, 2004.

[111] Q. Wei, W. Wei, B. Lai et al., "Uniform-sized PLA nanoparticles: preparation by premix membrane emulsification," International Journal of Pharmaceutics, vol. 359, no. 1-2, pp. 294-297, 2008.

[112] Y. Yu, S. Tan, S. Zhao et al., "Antitumor activity of docetaxelloaded polymeric nanoparticles fabricated by shirasu porous glass membrane-emulsification technique," International Journal of Nanomedicine, vol. 8, pp. 2641-2652, 2013.

[113] E. Imbuluzqueta, E. Elizondo, C. Gamazo et al., "Novel bioactive hydrophobic gentamicin carriers for the treatment of intracellular bacterial infections," Acta Biomaterialia, vol. 7, no. 4, pp. 1599-1608, 2011.
[114] E. Imbuluzqueta, C. Gamazo, H. Lana et al., "Hydrophobic gentamicin-loaded nanoparticles are effective against Brucella melitensis infection in mice," Antimicrobial Agents and Chemotherapy, vol. 57, no. 7, pp. 3326-3333, 2013.

[115] G. Dalwadi and B. Sunderland, "An ion pairing approach to increase the loading of hydrophilic and lipophilic drugs into PEGylated PLGA nanoparticles," European Journal of Pharmaceutics and Biopharmaceutics, vol. 71, no. 2, pp. 231-242, 2009.

[116] S. H. Choi and T. G. Park, "Hydrophobic ion pair formation between leuprolide and sodium oleate for sustained release from biodegradable polymeric microspheres," International Journal of Pharmaceutics, vol. 203, no. 1-2, pp. 193-202, 2000.

[117] L. Yang, F. Cui, K. Shi, D. Cun, and R. Wang, "Design of high payload PLGA nanoparticles containing melittinsodium dodecyl sulfate complex by the hydrophobic ion-pairing technique," Drug Development and Industrial Pharmacy, vol. 35, no. 8, pp. 959-968, 2009.

[118] F. Cui, K. Shi, L. Zhang, A. Tao, and Y. Kawashima, "Biodegradable nanoparticles loaded with insulin-phospholipid complex for oral delivery: preparation, in vitro characterization and in vivo evaluation," Journal of Controlled Release, vol. 114, no. 2, pp. 242-250, 2006.

[119] H. Kim, B.-J. Lee, and H. Sah, "Reverse micelle-based microencapsulation of oxytetracycline hydrochloride into poly- $d, l$ lactide-co-glycolide microspheres," Drug Delivery, vol. 14, no. 2, pp. 95-99, 2007.

[120] G. M. Whitesides, "The origins and the future of microfluidics," Nature, vol. 442, no. 7101, pp. 368-373, 2006.

[121] L. H. Hung and A. P. Lee, "Microfluidic devices for the synthesis of nanoparticles and biomaterials," Journal of Medical and Biological Engineering, vol. 27, no. 1, pp. 1-6, 2007.

[122] P. M. Valencia, O. C. Farokhzad, R. Karnik, and R. Langer, "Microfluidic technologies for accelerating the clinical translation of nanoparticles," Nature Nanotechnology, vol. 7, no. 10, pp. 623-629, 2012.

[123] A. Jahn, J. E. Reiner, W. N. Vreeland, D. L. DeVoe, L. E. Locascio, and M. Gaitan, "Preparation of nanoparticles by continuousflow microfluidics," Journal of Nanoparticle Research, vol. 10, no. 6, pp. 925-934, 2008.

[124] S. Marre and K. F. Jensen, "Synthesis of micro and nanostructures in microfluidic systems," Chemical Society Reviews, vol. 39, no. 3, pp. 1183-1202, 2010.

[125] L. Y. Yeo, H.-C. Chang, P. P. Y. Chan, and J. R. Friend, "Microfluidic devices for bioapplications," Small, vol. 7, no. 1, pp. 12-48, 2011.

[126] G. F. Christopher and S. L. Anna, "Microfluidic methods for generating continuous droplet streams," Journal of Physics D: Applied Physics, vol. 40, no. 19, pp. R319-R336, 2007.

[127] P. B. Umbanhowar, V. Prasad, and D. A. Weitz, "Monodisperse emulsion generation via drop break off in a colfowing stream," Langmuir, vol. 16, no. 2, pp. 347-351, 2000.

[128] H. Gu, M. H. G. Duits, and F. Mugele, "Droplets formation and merging in two-phase flow microfluidics," International Journal of Molecular Sciences, vol. 12, no. 12, pp. 2572-2597, 2011.

[129] T. Thorsen, R. W. Roberts, F. H. Arnold, and S. R. Quake, "Dynamic pattern formation in a vesicle-generating microfluidic device," Physical Review Letters, vol. 86, no. 18, pp. 41634166, 2001.

[130] H. Xie and J. W. Smith, "Fabrication of PLGA nanoparticles with a fluidic nanoprecipitation system," Journal of Nanobiotechnology, vol. 8, article 18, 2010. 
[131] B. K. Johnson and R. K. Prud'homme, "Flash Nano p recipitation of organic actives and block copolymers using a confined impinging jets mixer," Australian Journal of Chemistry, vol. 56, no. 10, pp. 1021-1024, 2003.

[132] Z. N. Wei, W. Li, M. Seo, S. Xu, and E. Kumacheva, "Janus and ternary particles generated by microfluidic synthesis: design, synthesis, and self-assembly," Journal of the American Chemical Society, vol. 128, no. 29, pp. 9408-9412, 2006.

[133] S. L. Anna, N. Bontoux, and H. A. Stone, "Formation of dispersions using 'flow focusing' in microchannels," Applied Physics Letters, vol. 82, no. 3, pp. 364-366, 2003.

[134] R. Dreyfus, P. Tabeling, and H. Willaime, "Ordered and disordered patterns in two-phase flows in microchannels," Physical Review Letters, vol. 90, no. 14, Article ID 144505, 2003.

[135] M. J. Cózar-Bernal, M. A. Holgado, J. L. Arias et al., "Insulinloaded PLGA microparticles: flow focusing versus double emulsion/solvent evaporation," Journal of Microencapsulation, vol. 28, no. 5, pp. 430-441, 2011.

[136] M. A. Holgado, M. J. Cózar-Bernal, S. Salas, J. L. Arias, J. Álvarez-Fuentes, and M. Fernández-Arévalo, "Protein-loaded PLGA microparticles engineered by flow focusing: physicochemical characterization and protein detection by reversedphase HPLC," International Journal of Pharmaceutics, vol. 380, no. 1-2, pp. 147-154, 2009.

[137] L. Martín-Banderas, M. Flores-Masquera, P. Riesco-Chueca et al., "Flow focusing: a versatile technology to produce sizecontrolled and specific-morphology microparticles," Small, vol. 1, no. 7, pp. 688-692, 2005.

[138] L. Martín-Banderas, E. Sáez-Fernández, M. Á. Holgado et al., "Biocompatible gemcitabine-based nanomedicine engineered by flow focusing for efficient antitumor activity," International Journal of Pharmaceutics, vol. 443, no. 1-2, pp. 103-109, 2013.

[139] R. Karnik, F. Gu, P. Basto et al., "Microfluidic platform for controlled synthesis of polymeric nanoparticles," Nano Letters, vol. 8, no. 9, pp. 2906-2912, 2008.

[140] J. M. Lim, N. Bertrand, P. M. Valencia et al., "Parallel microfluidic synthesis of size-tunable polymeric nanoparticles using 3D flow focusing towards in vivo study," Nanomedicine: Nanotechnology, Biology, and Medicine, vol. 10, no. 2, pp. 401-409, 2014.

[141] R. Krishnamurthy, J. A. Lumpkin, and R. Sridhar, "Inactivation of lysozyme by sonication under conditions relevant to microencapsulation," International Journal of Pharmaceutics, vol. 205, no. 1-2, pp. 23-34, 2000.

[142] C. Perez, A. Sanchez, D. Putnam, D. Ting, R. Langer, and M. J. Alonso, "Poly(lactic acid)-poly(ethylene glycol) nanoparticles as new carriers for the delivery of plasmid DNA," Journal of Controlled Release, vol. 75, no. 1-2, pp. 211-224, 2001.

[143] P. G. Shao and L. C. Bailey, "Porcine insulin biodegradable polyester microspheres: stability and in vitro release characteristics," Pharmaceutical Development and Technology, vol. 5, no. 1, pp. 1-9, 2000.

[144] M. A. Ibrahim, A. Ismail, M. I. Fetouh, and A. Göpferich, "Stability of insulin during the erosion of poly(lactic acid) and poly(lactic-co-glycolic acid) microspheres," Journal of Controlled Release, vol. 106, no. 3, pp. 241-252, 2005.

[145] G. Zhu, S. R. Mallery, and S. P. Schwendeman, "Stabilization of proteins encapsulated in injectable poly(lactide-co-glycolide)," Nature Biotechnology, vol. 18, no. 1, pp. 52-57, 2000.

[146] Y.-P. Li, Y.-Y. Pei, X.-Y. Zhang et al., "PEGylated PLGA nanoparticles as protein carriers: synthesis, preparation and biodistribution in rats," Journal of Controlled Release, vol. 71, no. 2, pp. 203-211, 2001.
[147] D. T. Martin, C. J. Hoimes, H. Z. Kaimakliotis et al., "Nanoparticles for urothelium penetration and delivery of the histone deacetylase inhibitor belinostat for treatment of bladder cancer," Nanomedicine: Nanotechnology, Biology, and Medicine, vol. 9, no. 8, pp. 1124-1134, 2013.

[148] S. S. Vasudev, S. Ahmad, R. Parveen et al., "Formulation of PEG-ylated L-asparaginase loaded poly(lactide-co-glycolide) nanoparticles: Influence of PEGylation on enzyme loading, activity and in vitro release," Pharmazie, vol. 66, no. 12, pp. 956960, 2011.

[149] J. X. Zhang and K. J. Zhu, "An improvement of double emulsion technique for preparing bovine serum albumin-loaded PLGA microspheres," Journal of Microencapsulation, vol. 21, no. 7, pp. 775-785, 2004.

[150] B. Kirthivasan, D. Singh, M. M. Bommana, S. L. Raut, E. Squillante, and M. Sadoqi, "Active brain targeting of a fluorescent P-gp substrate using polymeric magnetic nanocarrier system," Nanotechnology, vol. 23, no. 25, Article ID 255102, 2012.

[151] F. Ito, H. Fujimori, and K. Makino, "Factors affecting the loading efficiency of water-soluble drugs in PLGA microspheres," Colloids and Surfaces B: Biointerfaces, vol. 61, no. 1, pp. 25-29, 2008.

[152] L. Cheng, C. Jin, W. Lv, Q. Ding, and X. Han, "Developing a highly stable PLGA-mPEG nanoparticle loaded with cisplatin for chemotherapy of ovarian cancer," PLoS ONE, vol. 6, no. 9, Article ID e25433, 2011.

[153] P. D. Berkman and R. V. Calabrese, "dispersion of viscous liquids by turbulent flow in a static mixer," AIChE Journal, vol. 34, no. 4, pp. 602-609, 1988.

[154] R. K. Thakur, Ch. Vial, K. D. P. Nigam, E. B. Nauman, and G. Djelveh, "Static mixers in the process industries-a review," Chemical Engineering Research and Design, vol. 81, no. 7, pp. 787-826, 2003.

[155] T. Lemenand, D. D. Valle, Y. Zellouf, and H. Peerhossaini, "Droplets formation in turbulent mixing of two immiscible fluids in a new type of static mixer," International Journal of Multiphase Flow, vol. 29, no. 5, pp. 813-840, 2003.

[156] Y. F. Maa and C. Hsu, "Liquid-liquid emulsification by static mixers for use in microencapsulation," Journal of Microencapsulation, vol. 13, no. 4, pp. 419-433, 1996.

[157] S. I. Lyons and S. G. Wright, "Method and apparatus for preparing microparticles using in-line extraction," United States Patent US 6705757, Alkermes Controlled Therapeutic II, 2004.

[158] M. E. Rickey, M. Ramstack, and D. H. Lewis, "Preparation of biodegradable biocompatible microparticles containing a bioactive agent," United States Patent US 6403114, Alkermes Controlled Therapeutic II, 2002.

[159] S. Freitas, B. Rudolf, H. P. Merkle, and B. Gander, "Flow-through ultrasonic emulsification combined with static micromixing for aseptic production of microspheres by solvent extraction," European Journal of Pharmaceutics and Biopharmaceutics, vol. 61, no. 3, pp. 181-187, 2005.

[160] C. Wischke and H.-H. Borchert, "Influence of the primary emulsification procedure on the characteristics of small protein-loaded PLGA microparticles for antigen delivery," Journal of Microencapsulation, vol. 23, no. 4, pp. 435-448, 2006.

[161] Y. Dong, W. K. Ng, S. Shen, S. Kim, and R. B. H. Tan, "Solid lipid nanoparticles: continuous and potential large-scale nanoprecipitation production in static mixers," Colloids and Surfaces B: Biointerfaces, vol. 94, pp. 68-72, 2012.

[162] Y. Dong, W. K. Ng, S. Shen, S. Kim, and R. B. H. Tan, "Scalable ionic gelation synthesis of chitosan nanoparticles for drug 
delivery in static mixers," Carbohydrate Polymers, vol. 94, no. 2, pp. 940-945, 2013.

[163] J. Podobinski, M. Ramstack, and D. S. Dickey, "Methods and systems for generating nanoparticles," Inventors: Cerulean Pharma Inc. Assignee, US Patent no. 8618240, 2013.

[164] K. Ouzineb, C. Lord, N. Lesauze, C. Graillat, P. A. Tanguy, and T. McKenna, "Homogenisation devices for the production of miniemulsions," Chemical Engineering Science, vol. 61, no. 9, pp. 2994-3000, 2006.

[165] G. García del Barrio, J. Hendry, M. J. Renedo, J. M. Irache, and F. J. Novo, "In vivo sustained release of adenoviral vectors from poly(D,L-lactic-co-glycolic) acid microparticles prepared by TROMS," Journal of Controlled Release, vol. 94, no. 1, pp. 229$235,2004$.

[166] D. M. Santos, M. W. Carneiro, T. R. de Moura et al., "PLGA nanoparticles loaded with KMP-11 stimulate innate immunity and induce the killing of Leishmania," Nanomedicine, vol. 9, no. 7, pp. 985-995, 2013.

[167] Y. Liu, C. Cheng, R. K. Prud'homme, and R. O. Fox, "Mixing in a multi-inlet vortex mixer (MIVM) for flash nano-precipitation," Chemical Engineering Science, vol. 63, no. 11, pp. 2829-2842, 2008.

[168] Y. Liu, Z. Tong, and R. K. P. Homme, "Stabilized polymeric nanoparticles for controlled and efficient release of bifenthrin," Pest Management Science, vol. 64, no. 8, pp. 808-812, 2008.

[169] Y. Shi, J. C. Cheng, R. O. Fox, and M. G. Olsen, "Measurements of turbulence in a microscale multi-inlet vortex nanoprecipitation reactor," Journal of Micromechanics and Microengineering, vol. 23, no. 7, Article ID 075005, 2013.

[170] K. K. Cheng, C. F. Yeung, S. W. Ho, S. F. Chow, A. H. L. Chow, and L. Baum, "Highly stabilized curcumin nanoparticles tested in an in vitro blood-brain barrier model and in Alzheimer's disease Tg2576 Mice," The AAPS Journal, vol. 15, no. 2, pp. 324336, 2012.

[171] A. Aravind, S. H. Varghese, S. Veeranarayanan et al., "Aptamerlabeled PLGA nanoparticles for targeting cancer cells," Cancer Nanotechnology, vol. 3, no. 1-6, pp. 1-12, 2012.

[172] N. Schleich, P. Sibret, P. Danhier et al., "Dual anticancer drug/superparamagnetic iron oxide-loaded PLGA-based nanoparticles for cancer therapy and magnetic resonance imaging," International Journal of Pharmaceutics, vol. 447, no. 1-2, pp. 94-101, 2013.

[173] M. B. Heo and Y. T. Lim, "Programmed nanoparticles for combined immunomodulation, antigen presentation and tracking of immunotherapeutic cells," Biomaterials, vol. 35, no. 1, pp. 590-600, 2014.

[174] H. Wang, Y. Zhao, Y. Wu et al., "Enhanced anti-tumor efficacy by co-delivery of doxorubicin and paclitaxel with amphiphilic methoxy PEG-PLGA copolymer nanoparticles," Biomaterials, vol. 32, no. 32, pp. 8281-8290, 2011.

[175] X. Zhang, G. Chen, L. Wen et al., "Novel multiple agents loaded PLGA nanoparticles for brain delivery via inner ear administration: in vitro and in vivo evaluation," European Journal of Pharmaceutical Sciences, vol. 48, no. 4-5, pp. 595-603, 2013.

[176] Y. B. Patil, S. K. Swaminathan, T. Sadhukha, L. Ma, and J. Panyam, "The use of nanoparticle-mediated targeted gene silencing and drug delivery to overcome tumor drug resistance," Biomaterials, vol. 31, no. 2, pp. 358-365, 2010.

[177] Y. Cui, Q. Xu, P. K. H. Chow, D. Wang, and C. H. Wang, "Transferrin-conjugated magnetic silica PLGA nanoparticles loaded with doxorubicin and paclitaxel for brain glioma treatment," Biomaterials, vol. 34, no. 33, pp. 8511-8520, 2013.

[178] X. Yuan, S. Naguib, and Z. Wu, "Recent advances of siRNA delivery by nanoparticles," Expert Opinion on Drug Delivery, vol. 8, no. 4, pp. 521-536, 2011.

[179] M. V. Yezhelyev, L. Qi, R. M. O’Regan, S. Nie, and X. Gao, "Proton-sponge coated quantum dots for siRNA delivery and intracellular imaging," Journal of the American Chemical Society, vol. 130, no. 28, pp. 9006-9012, 2008.

[180] Y. Zhou, L. Zhang, W. Zhao, Y. Wu, C. Zhu, and Y. Yang, "Nanoparticle-mediated delivery of TGF- $\beta 1$ miRNA plasmid for preventing flexor tendon adhesion formation," Biomaterials, vol. 34, no. 33, pp. 8269-8278, 2013.

[181] Y. Wang, S. Gao, W.-H. Ye, H. S. Yoon, and Y.-Y. Yang, "Codelivery of drugs and DNA from cationic core-shell nanoparticles self-assembled from a biodegradable copolymer," Nature Materials, vol. 5, no. 10, pp. 791-796, 2006.

[182] X. Yuan, B. A. Shah, N. K. Kotadia, J. Li, H. Gu, and Z. $\mathrm{Wu}$, "The development and mechanism studies of cationic chitosan-modified biodegradable PLGA nanoparticles for efficient siRNA drug delivery," Pharmaceutical Research, vol. 27, no. 7, pp. 1285-1295, 2010.

[183] D. N. Nguyen, J. J. Green, J. M. Chan, R. Langer, and D. G. Anderson, "Polymeric materials for gene delivery and DNA vaccination," Advanced Materials, vol. 21, no. 8, pp. 847-867, 2009.

[184] F. Ito, Y. Uchida, and Y. Murakami, "Facile technique for preparing organic-inorganic composite particles: monodisperse poly(lactide-co-glycolide) (PLGA) particles having silica nanoparticles on the surface," Colloids and Surfaces A: Physicochemical and Engineering Aspects, vol. 361, no. 1-3, pp. 109-117, 2010.

[185] J. M. Chan, L. Zhang, K. P. Yuet et al., "PLGA-lecithin-PEG coreshell nanoparticles for controlled drug delivery," Biomaterials, vol. 30, no. 8, pp. 1627-1634, 2009.

[186] G. Ling, P. Zhang, W. Zhang et al., "Development of novel selfassembled DS-PLGA hybrid nanoparticles for improving oral bioavailability of vincristine sulfate by P-gp inhibition," Journal of Controlled Release, vol. 148, no. 2, pp. 241-248, 2010.

[187] B. Mandal, H. Bhattacharjee, N. Mittal et al., "Core-shell-type lipid-polymer hybrid nanoparticles as a drug delivery platform," Nanomedicine: Nanotechnology, Biology, and Medicine, vol. 9, no. 4, pp. 474-491, 2013.

[188] M. Zheng, C. Yue, Y. Ma et al., "Single-step assembly of DOX/ICG loaded lipid-polymer nanoparticles for highly effective chemo-photothermal combination therapy," ACS Nano, vol. 7, no. 3, pp. 2056-2067, 2013.

[189] S. Sengupta, D. Eavarone, I. Capila et al., "Temporal targeting of tumour cells and neovasculature with a nanoscale delivery system," Nature, vol. 436, no. 7050, pp. 568-572, 2005.

[190] F. Huang, M. You, T. Chen, G. Zhu, H. Liang, and W. Tan, "Selfassembled hybrid nanoparticles for targeted co-delivery of two drugs into cancer cells," Chemical Communications, vol. 50, no. 23, pp. 3103-3105, 2014.

[191] Y. Wu and R. L. Clark, "Electrohydrodynamic atomization: a versatile process for preparing materials for biomedical applications," Journal of Biomaterials Science, Polymer Edition, vol. 19, no. 5, pp. 573-601, 2008.

[192] S. Labbaf, S. Deb, G. Cama, E. Stride, and M. Edirisinghe, "Preparation of multicompartment sub-micron particles using a triple-needle electrohydrodynamic device," Journal of Colloid and Interface Science, vol. 409, pp. 245-254, 2013. 
[193] M. C. Branco and J. P. Schneider, "Self-assembling materials for therapeutic delivery," Acta Biomaterialia, vol. 5, no. 3, pp. 817831, 2009.

[194] F. Ahmed, R. I. Pakunlu, A. Brannan, F. Bates, T. Minko, and D. E. Discher, "Biodegradable polymersomes loaded with both paclitaxel and doxorubicin permeate and shrink tumors, inducing apoptosis in proportion to accumulated drug," Journal of Controlled Release, vol. 116, no. 2, pp. 150-158, 2006.

[195] Y. Zhang, H. F. Chan, and K. W. Leong, "Advanced materials and processing for drug delivery: the past and the future," Advanced Drug Delivery Reviews, vol. 65, no. 1, pp. 104-120, 2013.

[196] "ICH Harmonized Tripartite Guideline-Impurities: Guideline for residual solvents Q3C(R5)," March 2010.

[197] J. Herrmann and R. Bodmeier, "Biodegradable, somatostatin acetate containing microspheres prepared by various aqueous and non-aqueous solvent evaporation methods," European Journal of Pharmaceutics and Biopharmaceutics, vol. 45, no. 1, pp. 75-82, 1998.

[198] F. J. Wang and C. H. Wang, "Sustained release of etanidazole from spray dried microspheres prepared by non-halogenated solvents," Journal of Controlled Release, vol. 81, no. 3, pp. 263280, 2002.

[199] I. J. Castellanos and K. Griebenow, "Improved $\alpha$-chymotrypsin stability upon encapsulation in PLGA microspheres by solvent replacement," Pharmaceutical Research, vol. 20, no. 11, pp. 18731880, 2003.

[200] F. T. Meng, G. H. Ma, W. Qiu, and Z. G. Su, "W/O/W double emulsion technique using ethyl acetate as organic solvent: effects of its diffusion rate on the characteristics of microparticles," Journal of Controlled Release, vol. 91, no. 3, pp. 407-416, 2003.

[201] Y. Yeo, A. U. T. Chen, O. A. Basaran, and K. Park, "Microencapsulation of drug by solvent exchange," United States patent US 6599627, Purdue Research Fouondation, West Lafayette, Ind, USA, 2003.

[202] A. Matsumoto, T. Kitazawa, J. Murata, Y. Horikiri, and H. Yamahara, "A novel preparation method for PLGA microspheres using non-halogenated solvents," Journal of Controlled Release, vol. 129 , no. 3, pp. 223-227, 2008.

[203] H. Hamishehkar, J. Emami, A. R. Najafabadi et al., "The effect of formulation variables on the characteristics of insulin-loaded poly(lactic-co-glycolic acid) microspheres prepared by a single phase oil in oil solvent evaporation method," Colloids and Surfaces B: Biointerfaces, vol. 74, no. 1, pp. 340-349, 2009.

[204] H.-Y. Im and H. Sah, "Ammonolysis-based microencapsulation technique using isopropyl formate as dispersed solvent," International Journal of Pharmaceutics, vol. 382, no. 1-2, pp. 130-138, 2009.

[205] W. M. Obeidat, "Recent patents review in microencapsulation of pharmaceuticals using the emulsion solvent removal methods," Recent Patents on Drug Delivery and Formulation, vol. 3, no. 3, pp. 178-192, 2009.

[206] D. Allhenn and A. Lamprecht, "Microsphere preparation using the untoxic solvent glycofurol," Pharmaceutical Research, vol. 28, no. 3, pp. 563-571, 2011.

[207] D. T. Birnbaum, J. D. Kosmala, D. B. Henthorn, and L. Brannon-Peppas, "Controlled release of $\beta$-estradiol from PLAGA microparticles: the effect of organic phase solvent on encapsulation and release," Journal of Controlled Release, vol. 65, no. 3, pp. 375-387, 2000.

[208] M. Li, O. Rouaud, and D. Poncelet, "Microencapsulation by solvent evaporation: state of the art for process engineering approaches,' International Journal of Pharmaceutics, vol. 363, no. 1-2, pp. 26-39, 2008.

[209] C. B'Hymer, "Residual solvent testing: a review of gaschromatographic and alternative techniques," Pharmaceutical Research, vol. 20, no. 3, pp. 337-344, 2003.

[210] N. Desai, "Challenges in development of nanoparticle-based therapeutics," AAPS Journal, vol. 14, no. 2, pp. 282-295, 2012.

[211] I. P. Kaur, V. Kakkar, P. K. Deol, M. Yadav, M. Singh, and I. Sharma, "Issues and concerns in nanotech product development and its commercialization," Journal of Controlled Release, vol. 193, pp. 51-62, 2014. 

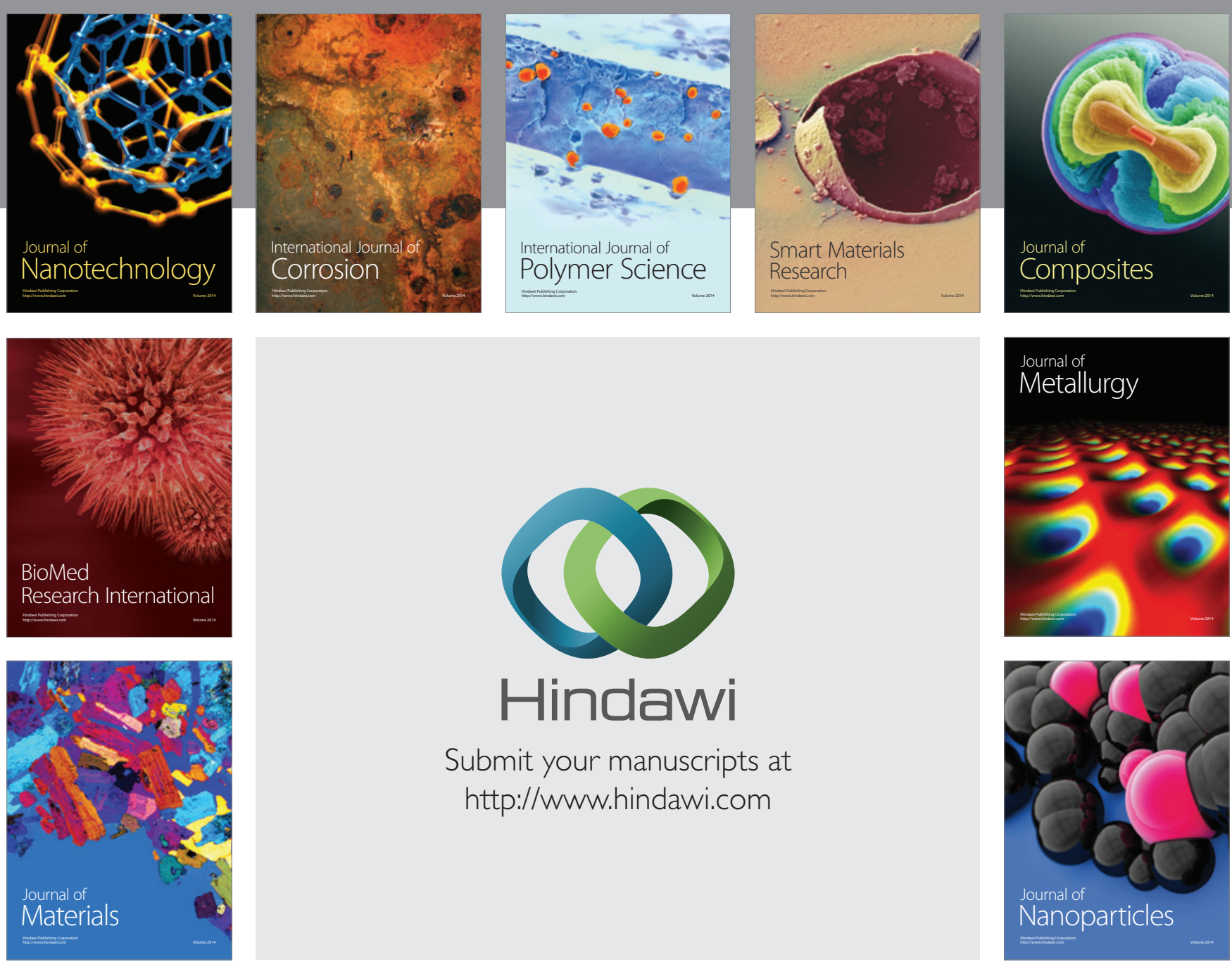

Submit your manuscripts at http://www.hindawi.com
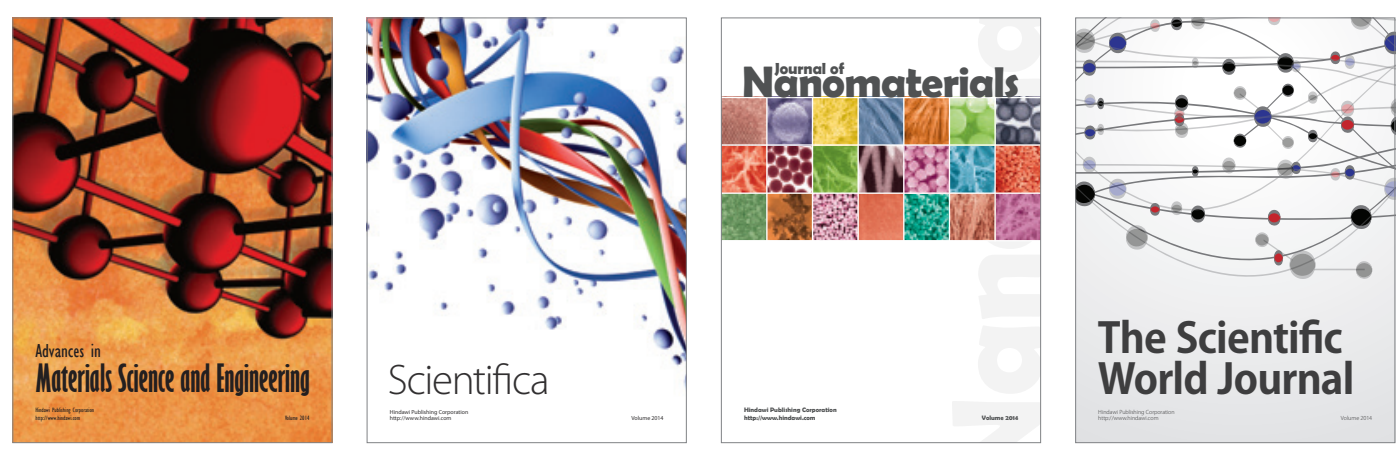

\section{The Scientific World Journal}
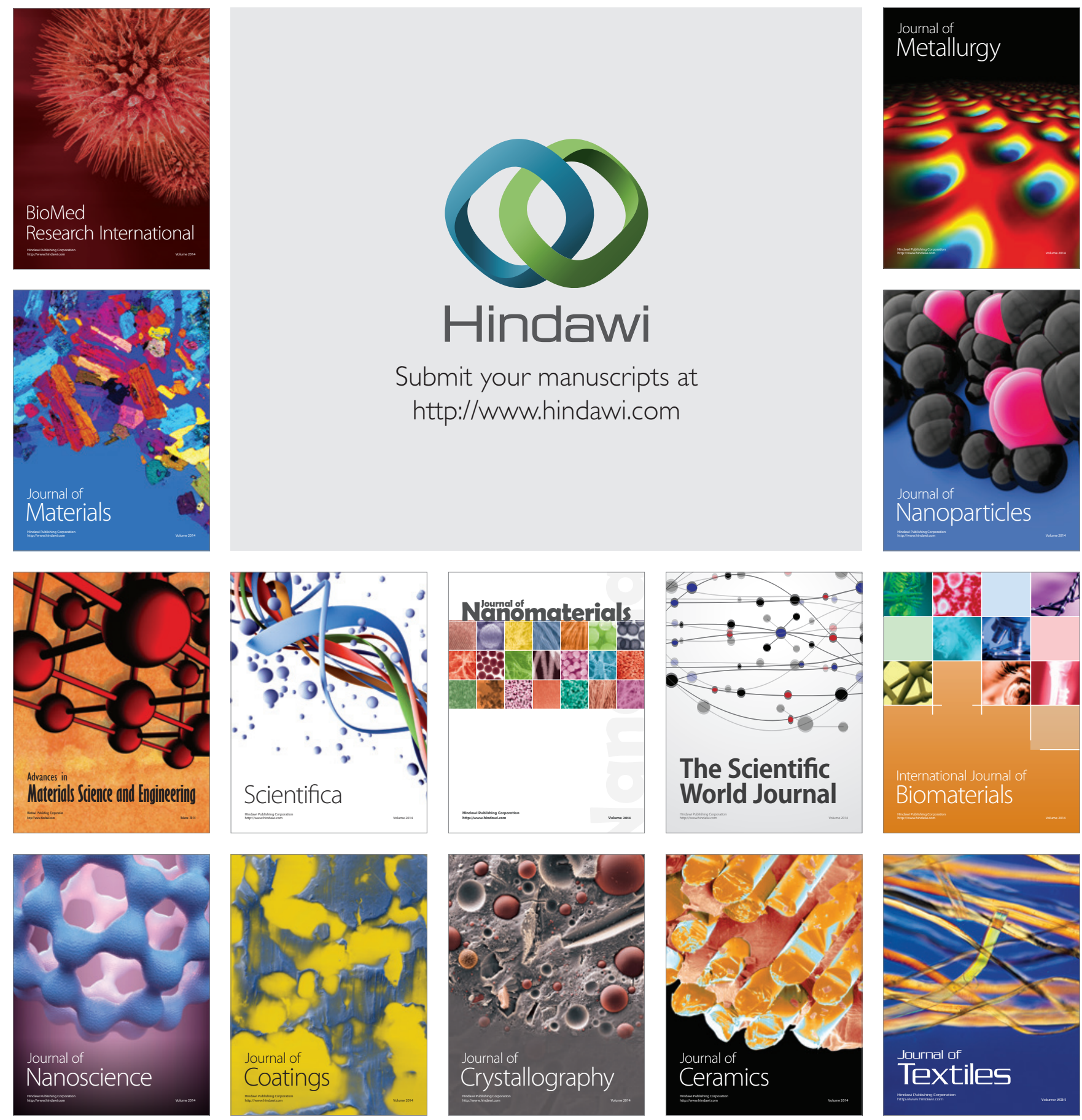